
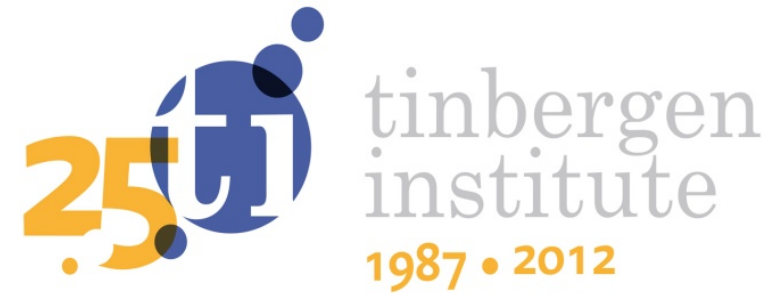

\title{
Joint Independent Metropolis-Hastings Methods for Nonlinear Non-Gaussian State Space Models
}

\author{
Istvan Barra* \\ Lennart Hoogerheide \\ Siem Jan Koopman \\ André Lucas
}

Faculty of Economics and Business Administration, VU University Amsterdam, and Tinbergen Institute.

*Duisenberg School of Finance 
Tinbergen Institute is the graduate school and research institute in economics of Erasmus University Rotterdam, the University of Amsterdam and VU University Amsterdam.

More TI discussion papers can be downloaded at http://www.tinbergen.nl

Tinbergen Institute has two locations:

Tinbergen Institute Amsterdam

Gustav Mahlerplein 117

1082 MS Amsterdam

The Netherlands

Tel.: +31(0)205251600

Tinbergen Institute Rotterdam

Burg. Oudlaan 50

3062 PA Rotterdam

The Netherlands

Tel.: +31(0)10 4088900

Fax: $+31(0) 104089031$

Duisenberg school of finance is a collaboration of the Dutch financial sector and universities, with the ambition to support innovative research and offer top quality academic education in core areas of finance.

DSF research papers can be downloaded at: http://www.dsf.nl/

Duisenberg school of finance

Gustav Mahlerplein 117

1082 MS Amsterdam

The Netherlands

Tel.: +31(0)20 5258579 


\title{
Joint Independent Metropolis-Hastings Methods for Nonlinear Non-Gaussian State Space Models *
}

\author{
István Barra, ${ }^{(a, b)}$ Lennart Hoogerheide, ${ }^{(a), \ddagger}$ Siem Jan Koopman, ${ }^{(a)}$ André Lucas ${ }^{(a)}$ \\ (a) VU University Amsterdam, and Tinbergen Institute \\ (b) Duisenberg School of Finance
}

March 24, 2013

\begin{abstract}
We propose a new methodology for the Bayesian analysis of nonlinear nonGaussian state space models with a Gaussian time-varying signal, where the signal is a function of a possibly high-dimensional state vector. The novelty of our approach is the development of proposal densities for the joint posterior density of parameter and state vectors: a mixture of Student's $t$-densities as the marginal proposal density for the parameter vector, and a Gaussian density as the conditional proposal density for the signal given the parameter vector. We argue that a highly efficient procedure emerges when these proposal densities are used in an independent Metropolis-Hastings algorithm. A particular feature of our approach is that smoothed estimates of the states and an estimate of the marginal likelihood are obtained directly as an output of the algorithm. Our methods are computationally efficient and produce more accurate estimates when compared to recently proposed alternatives. We present extensive simulation evidence for stochastic volatility and stochastic intensity models. For our empirical study, we analyse the performance of our method for stock return data and corporate default panel data.
\end{abstract}

JEL classification: C11, C15, C22, C32, C58.

Keywords: nonlinear non-Gaussian state space model, Bayesian inference, Monte Carlo estimation, Metropolis-Hastings algorithm, mixture of Student's $t$-distributions.

*Author information: István Barra, E-mail: i.barra@vu.nl. Lennart Hoogerheide , E-mail: l.f.hoogerheide@vu.nl. Siem Jan Koopman, E-mail: s.j.koopman@vu.nl . André Lucas, Email:a.lucas@vu.nl. Address: VU University Amsterdam, De Boelelaan 1105, 1081 HV.

$\ddagger$ Corresponding author. 


\section{Introduction}

Empirical studies based on nonlinear non-Gaussian state space models are widespread in economics and finance. It is well known that the Bayesian estimation of parameters and states can be difficult. In particular, the evaluation of the marginal likelihood function is a challenging task. One of the complications is that the joint posterior density of the parameter and state vectors is typically high-dimensional which makes it cumbersome to develop successful proposal distributions and Monte Carlo algorithms. It is standard practice to overcome this difficulty by disentangling the target density into lower dimensional densities and develop proposal densities for each of them. However, this approach leads to other problems. Although the curse of dimensionality may be resolved to some extent, it is rather demanding to design a proposal density on a case by case basis for each lower dimensional target density. Furthermore, these separately defined proposal densities may not adequately characterize the properties of the joint posterior density, possibly resulting in unsatisfactory computational performance of the method and to biased estimates of posterior moments and marginal likelihoods.

The aim of our paper is to develop an independent Metropolis-Hastings (MH) procedure to sample from the joint posterior density of the parameters and states. The proposal density in our Joint Independent Metropolis-Hastings method consists of two components: (i) a mixture of Student's $t$-densities that targets the marginal posterior density of the parameters, and (ii) an approximating density that targets the density of the states given the observations and the parameters. The mixture of Student's $t$-densities is constructed by means of the Mixture of $t$ by Importance Sampling weighted Expectation Maximization (MitISEM) method of Hoogerheide et al. (2012). The proposal density for the states is then based on a given set of parameters. For this, we can take any reasonable approximating density for the states including those developed by Shephard and Pitt (1997), Durbin and Koopman (1997), Richard and Zhang (2007), Koopman et al. (2011) and McCausland (2012). We can use the proposal densities in an independent MH algorithm or in an importance sampling procedure to estimate the marginal likelihood and parameters. The resulting procedure can be almost fully automated and requires no user intervention.

We argue and show that our approach is computationally efficient and robust and can be regarded as an effective alternative to existing Markov chain Monte Carlo (MCMC) methods. Our method provides at least two advantages. First, the methodology can be fully automated. There is no need for case by case fine tuning of the algorithm whenever a different model specification with a possibly different observation density is considered. Second, the necessary computations can be implemented in a parallel manner. This 
implies that we can use state-of-the-art computer technology based on graphics cards to further reduce the computing time of our method.

In an extensive simulation study we show that our Joint Independent MetropolisHastings method is a viable alternative to the Adaptive Independent Metropolis-Hastings method as recently proposed by Pitt et al. (2012). We compare the methods in detail for two cases: the stochastic volatility model and the stochastic intensity model. In our Monte Carlo study, we find that our new method is computationally faster than the recently developed particle filter MCMC methods of Andrieu et al. (2010). In particular, our method provides posterior draws and estimates of posterior moments in a computationally more efficient manner.

Our work relates to two strands in the literature. First, we contribute to the more recent literature on Bayesian estimation of nonlinear non-Gaussian state space models by jointly sampling parameters and state paths. McCausland (2012) suggests a proposal density based on a higher order approximation of the states given the parameter vector. Although his sampler appears to be efficient, it relies on the assumption that the state vector is univariate. Chan and Strachan (2012) propose a method that overcomes this restriction. Their proposal density for the state vectors, however, is derived from a local approximation of the smoothed density, which can lead to poor performance in higher dimensional problems.

Second, our paper relates to the literature on the Bayesian estimation of nonlinear non-Gaussian state space models using particle filters. Andrieu et al. (2010) develop a collection of Particle Markov Chain Monte Carlo (PMCMC) methods for parameter estimation. As argued by Flury and Shephard (2011), the key idea of PMCMC methods is that the unknown true likelihood can be replaced by an unbiased estimator of the likelihood within any MCMC procedure. Although PMCMC methods provide a general solution to parameter and state estimation in nonlinear non-Gaussian state space models, they require the application of a particle filter for each iteration, see for example Doucet et al. (2012). To overcome this computational burden, Lindsten and Schon (2012) propose a modified version of the particle Gibbs sampler. For the same motivation, Pitt et al. (2012) develop an adaptive version of the particle independent Metropolis-Hastings algorithm with partially adapted auxiliary particle filters. We show in our simulations that in settings of empirical interest, particle filters may not always be the most efficient or robust approach from a numerical perspective.

The remainder of this paper is organized as follows. In Section 2 we introduce the new methodology. In Section 3 we demonstrate the performance of the methodology against state-of-the-art alternatives in a Monte Carlo study designed for parameter and 
state estimation in stochastic volatility and stochastic intensity models. In Section 4, we empirically illustrate the methods by considering a long time series of IBM stock returns and a large panel data set of U.S. corporate defaults. Section 5 concludes.

\section{Joint independent Metropolis-Hastings method}

For a time series of observations $y_{1}, \ldots, y_{T}$, we define the nonlinear non-Gaussian state space model by the observation density and the state equation

$$
\begin{gathered}
y_{t} \sim p_{y}\left(y_{t} \mid x_{t} ; \theta\right), \quad x_{t}=c_{t}+Z_{t} \alpha_{t}, \\
\alpha_{t+1}=d_{t}+T_{t} \alpha_{t}+\eta_{t}, \quad \eta_{t} \sim \mathrm{N}\left(0, Q_{t}\right),
\end{gathered}
$$

where $p_{y}$ is the observation density, $x_{t}$ is the signal, $\theta$ is the parameter vector, and $\alpha_{t}$ is the state vector, for $t=1, \ldots, T$. For expositional purposes, we assume that the observation $y_{t}$ and the signal $x_{t}$ are scalars. We discuss generalizations in Section 2.4. The signal is a linear function of the state vector $\alpha_{t}$, with scalar intercept $c_{t}$ and loading vector $Z_{t}$ both possibly depending in a deterministic way on time and on the parameter vector $\theta$, we have $c_{t}=c(t ; \theta)$ and $Z_{t}=Z(t ; \theta)$. The state vector $\alpha_{t}$ evolves as a linear Gaussian dynamic process given by (2) where the intercept vector $d_{t}=d(t ; \theta)$, transition matrix $T_{t}=T(t ; \theta)$ and variance matrix $Q_{t}=Q(t ; \theta)$ are deterministic functions of $t$ and $\theta$. We assume that all vectors and matrices have appropriate dimensions. Bayesian inference for model (1) and (2) involves the estimation of the properties of interest of the posterior density $p(\theta \mid y)$ of the parameter vector $\theta$ and the smoothed density of the signal $p(x \mid y)$, where $y=\left(y_{1}, \ldots, y_{T}\right)^{\prime}$ and $x=\left(x_{1}, \ldots, x_{T}\right)^{\prime}$.

\subsection{Independent Metropolis-Hastings}

We base our analysis on the development of an independent Metropolis-Hastings sampler; see Metropolis et al. (1953) and Hastings (1970) for the original contributions. We draw from the joint posterior density of the parameters and states $p(x, \theta \mid y)$. Our procedure consists of two phases: the training phase and the Markov chain Monte Carlo (MCMC) phase.

In the training phase we construct a proposal density that approximates the joint posterior $p(x, \theta \mid y)$. We construct the approximation from proposal densities $q(x \mid \theta, y)$ and $q_{\zeta}(\theta \mid y)$, where $q(x \mid \theta, y)$ is the conditional proposal density of $x$ given $\theta$, and $q_{\zeta}(\theta \mid y)$ is the marginal proposal density for $\theta$, where $\zeta$ refers to a set of auxiliary parameters. We 
approximate the target density $p(x \mid y ; \theta) p(\theta \mid y)$ well if $q(x \mid \theta, y)$ and $q_{\zeta}(\theta \mid y)$ are sufficiently close to $p(x \mid y ; \theta)$ and $p(\theta \mid y)$, respectively. We take $q_{\zeta}(\theta \mid y)$ as a mixture of Student's $t$ densities and use it to approximate $p(\theta \mid y)$, where $\zeta$ includes mode vectors, scale matrices, degrees of freedom and mixing weights for the Student's $t$-distributions in the candidate mixture $q_{\zeta}(\theta \mid y)$; see Hoogerheide et al. (2012) for further details. We take $q(x \mid \theta, y)$ as a Gaussian density following the numerically accelerated importance sampling (NAIS) method of Koopman et al. (2011), which is argued to be numerically more efficient than alternative approximations as suggested by Richard and Zhang (2007), Shephard and Pitt (1997), or Durbin and Koopman (1997).

In the Markov chain Monte Carlo phase we use the candidate as the proposal density in an independent Metropolis-Hastings algorithm to draw from the joint posterior density $p(x, \theta \mid y)$. We sample the joint candidate draws $\left(\theta^{(j)}, x^{(j)}\right)$ by first sampling

$$
\theta^{(j)} \sim q_{\zeta}(\theta \mid y)
$$

and then, conditioning on $\theta^{(j)}$, sampling

$$
x^{(j)} \sim q\left(x \mid \theta^{(j)}, y\right) .
$$

Let $\left(\theta^{(i-1)}, x^{(i-1)}\right)$ and $\left(\theta^{+}, x^{+}\right)$denote the previous accepted draw of the Markov chain and the new candidate draw, respectively. We set $\left(\theta^{(i)}, x^{(i)}\right)=\left(\theta^{+}, x^{+}\right)$with probability

$$
\alpha=\min \left\{\frac{p\left(\theta^{+}, x^{+} \mid y\right) q\left(x^{(i-1)} \mid \theta^{(i-1)}, y\right) q_{\zeta}\left(\theta^{(i-1)} \mid y\right)}{p\left(\theta^{(i-1)}, x^{(i-1)} \mid y\right) q\left(x^{+} \mid \theta^{+}, y\right) q_{\zeta}\left(\theta^{+} \mid y\right)}, 1\right\}
$$

and $\left(\theta^{(i)}, x^{(i)}\right)=\left(\theta^{(i-1)}, x^{(i-1)}\right)$ otherwise.

\subsection{A novel modification of MitISEM for nonlinear non-Gaussian state space models}

In the original MitISEM procedure, Hoogerheide et al. (2012) propose to approximate the posterior density $p(\theta \mid y)$ (of which only a kernel is required) by considering the Student's $t$ mixture $q_{\zeta}(\theta \mid y)$ and by minimizing the Kullback and Leibler (1951) divergence

$$
\int p(\theta \mid y) \log p(\theta \mid y) \mathrm{d} \theta-\int p(\theta \mid y) \log q_{\zeta}(\theta \mid y) \mathrm{d} \theta
$$

Since the first term does not depend on the proposal, an approximation of the KullbackLeibler divergence can be minimized by maximizing

$$
\frac{1}{N} \sum_{j=1}^{N} \frac{p\left(\theta^{(j)} \mid y\right)}{q_{0}\left(\theta^{(j)} \mid y\right)} \log q_{\zeta}\left(\theta^{(j)} \mid y\right)
$$


where $\theta^{(j)} \sim q_{0}(\theta \mid y)$ is an independent and identically distributed (i.i.d) sequence for $j=1, \ldots, N$. Unfortunately, in nonlinear non-Gaussian state space models we do not know (a kernel of) the posterior density $p\left(\theta^{(j)} \mid y\right)$ in closed form, so that we have to modify the original MitISEM method.

The Kullback-Leibler divergence between our target density $p(x, \theta \mid y)$ and the joint proposal density $q_{\zeta}(x, \theta \mid y)$ is given by

$$
\int p(x, \theta \mid y) \log p(x, \theta \mid y) \mathrm{d} x \mathrm{~d} \theta-\int p(x, \theta \mid y) \log q_{\zeta}(x, \theta \mid y) \mathrm{d} x \mathrm{~d} \theta .
$$

Minimizing the KL divergence is therefore equivalent to maximizing

$$
\int p(x, \theta \mid y) \log q_{\zeta}(x, \theta \mid y) \mathrm{d} x \mathrm{~d} \theta=\int \frac{p(x, \theta \mid y)}{q_{0}(x, \theta \mid y)} q_{0}(x, \theta \mid y) \log q_{\zeta}(x, \theta \mid y) \mathrm{d} x \mathrm{~d} \theta,
$$

where $q_{0}(x, \theta \mid y)$ is a previous candidate, which is used as an importance density. By decomposing the joint density $q_{\zeta}(x, \theta \mid y)=q(x \mid \theta, y) q_{\zeta}(\theta \mid y)$, where $q(x \mid \theta, y)$ is obtained using the NAIS method, we obtain

$$
\int \frac{p(x, \theta \mid y)}{q_{0}(x, \theta \mid y)} q_{0}(x, \theta \mid y) \log q(x \mid \theta, y) \mathrm{d} x \mathrm{~d} \theta+\int \frac{p(x, \theta \mid y)}{q_{0}(x, \theta \mid y)} q_{0}(x, \theta \mid y) \log q_{\zeta}(\theta \mid y) \mathrm{d} x \mathrm{~d} \theta .
$$

The first term in (10) does not depend on $\zeta$. Hence we maximize the second term in (10) that is approximated by

$$
\int \frac{p(x, \theta \mid y)}{q_{0}(x, \theta \mid y)} q_{0}(x, \theta \mid y) \log q_{\zeta}(\theta \mid y) \mathrm{d} x \mathrm{~d} \theta \approx \frac{1}{N} \sum_{j=1}^{N} \frac{p\left(x^{(j)}, \theta^{(j)} \mid y\right)}{q_{0}\left(x^{(j)}, \theta^{(j)} \mid y\right)} \log q_{\zeta}\left(\theta^{(j)} \mid y\right),
$$

where $\left(x^{(j)}, \theta^{(j)}\right) \sim q_{0}(x, \theta \mid y)$ is an i.i.d. sequence for $j=1, \ldots, N$.

If we compare (7) with (11), we see that the only difference with the original MitISEM approach is that we replace the weight $p\left(\theta^{(j)} \mid y\right) / q_{0}\left(\theta^{(j)} \mid y\right)$ by $p\left(x^{(j)}, \theta^{(j)} \mid y\right) / q_{0}\left(x^{(j)}, \theta^{(j)} \mid y\right)$. This novel result implies that we can use the MitISEM algorithm as described in Appendix A, with only a slight modification. The new weights

$$
w^{(j)}=\frac{p\left(x^{(j)}, \theta^{(j)} \mid y\right)}{q\left(x^{(j)} \mid \theta^{(j)}, y\right) q_{\zeta}\left(\theta^{(j)} \mid y\right)} \propto \frac{p\left(y \mid x^{(j)}, \theta^{(j)}\right) p\left(x^{(j)} \mid \theta^{(j)}\right) p\left(\theta^{(j)}\right)}{q\left(x^{(j)} \mid \theta^{(j)}, y\right) q_{\zeta}\left(\theta^{(j)} \mid y\right)}
$$

can be replaced by

$$
w^{(j)} \propto q\left(y \mid \theta^{(j)}\right) \frac{p\left(y \mid x^{(j)}, \theta^{(j)}\right) p\left(\theta^{(j)}\right)}{q\left(y \mid x^{(j)}, \theta^{(j)}\right) q_{\zeta}\left(\theta^{(j)} \mid y\right)},
$$

where we used the relations

$$
q\left(x^{(j)} \mid \theta^{(j)}, y\right)=\frac{q\left(y \mid x^{(j)}, \theta^{(j)}\right) q\left(x^{(j)} \mid \theta^{(j)}\right)}{q\left(y \mid \theta^{(j)}\right)},
$$

and $p\left(x^{(j)} \mid \theta^{(j)}\right)=q\left(x^{(j)} \mid \theta^{(j)}\right)$, since we have the same Gaussian linear state equation in the true model and the approximating Gaussian linear state space model upon which our 
Figure 1: Flow diagram of the joint independent Metropolis-Hastings (JIMH) procedure.

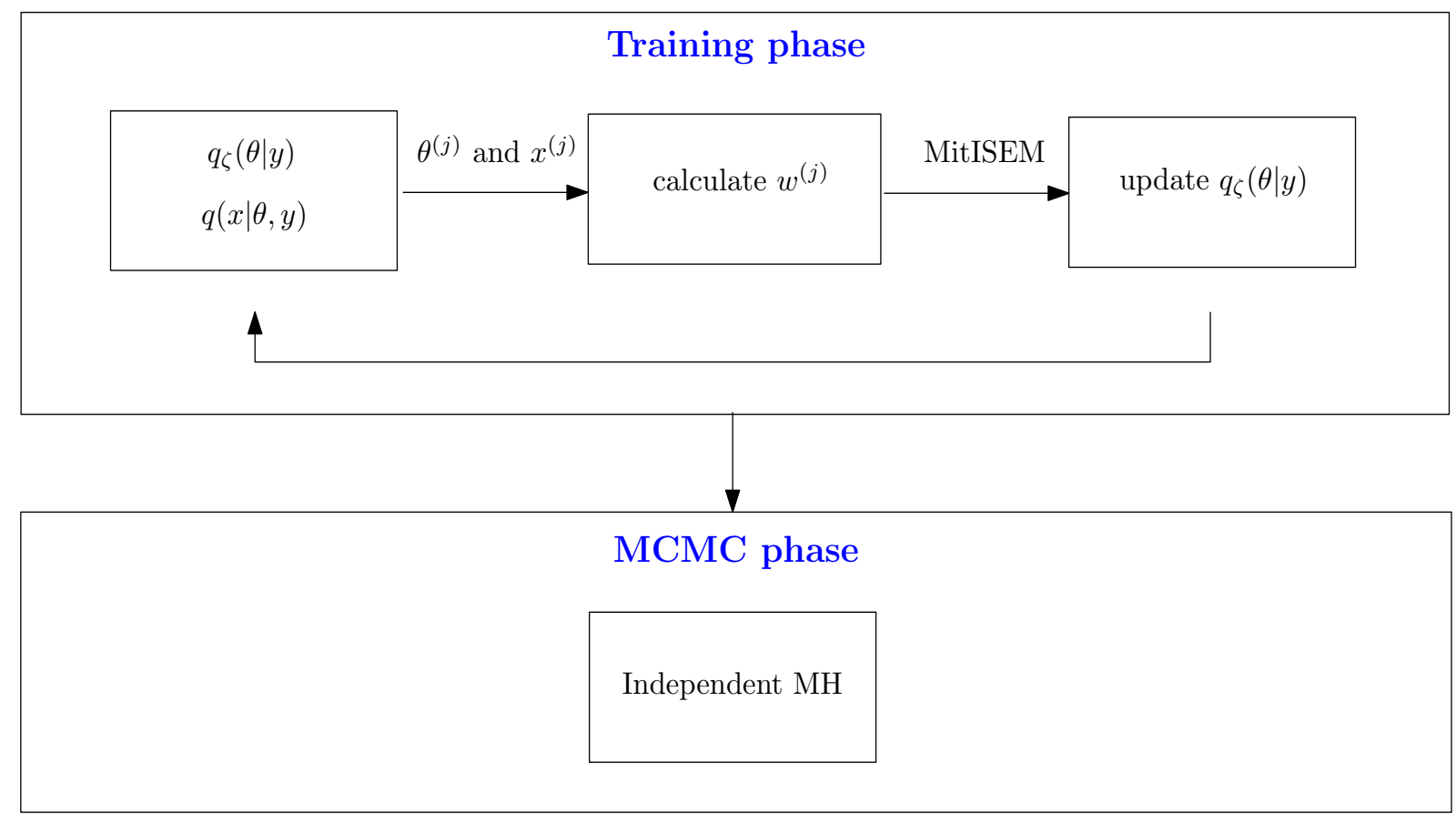

proposal density for the signal is based, see Appendix B for the details on this approximating model and the proposal density for the signal. The formulation in (13) is more convenient than (12), as we do not have to evaluate the density $q\left(x^{(j)} \mid \theta^{(j)}, y\right)$.

\subsection{The joint independent Metropolis-Hastings algorithm}

We present a summary of our newly proposed Joint Independent Metropolis-Hastings $(\mathrm{JIMH})$ algorithm in the flow-diagram of Figure 1. The algorithm can be described in more detail by the following steps.

\section{Training phase:}

(a) Initialization: Simulate a series of $N$ parameter vector draws $\theta^{(1)}, \ldots, \theta^{(N)}$ from a Student's $t$ distribution with its mode equal to the simulated maximum likelihood estimate of $\theta$ and with its scale equal to minus the inverse Hessian of the log likelihood evaluated at the current parameter estimates. We refer to this initial density as $q_{\zeta_{0}}$. Conditionally on the draws $\theta^{(1)}, \ldots, \theta^{(N)}$ we simulate a corresponding series of $N$ signal paths for $x$ from $q\left(x \mid \theta^{(j)}, y\right)$ and denote these by $x^{(1)}, \ldots, x^{(N)}$. Finally, we evaluate the joint importance sampling (IS) weights $w^{(1)}, \ldots, w^{(N)}$ given by (13). 
(b) Adaptation: Estimate the mean and variance of the target distribution via IS using the draws from $q_{\zeta_{0}}$. We adopt the estimated mean and variance as the mode and scale of the new proposal distribution that we denote by $q_{\zeta_{0}^{a}}$. We simulate draws $\theta^{(1)}, \ldots, \theta^{(N)}$ from $q_{\zeta_{0}^{a}}$. Finally we simulate signal paths $x^{(1)}, \ldots, x^{(N)}$ conditionally on the parameter draws, and evaluate the joint IS weights $w^{(1)}, \ldots, w^{(N)}$ given by (13).

(c) IS weighted EM algorithm: We obtain the updated proposal $q_{\zeta}$ from the IS weighted EM algorithm of MitISEM, using the latest draws and corresponding IS weights in (13). Appendix A provides further details about the MitISEM algorithm. We simulate draws $\theta^{(1)}, \ldots, \theta^{(N)}$ from the updated proposal $q_{\zeta}$, and signal paths $x^{(1)}, \ldots, x^{(N)}$ conditionally on these parameter draws. We compute the corresponding IS weights $w^{(1)}, \ldots, w^{(N)}$ in (13).

(d) Iterate on the number of mixture components: We now consider $10 \%$ of the simulated draws that corresponds to the highest IS weights, based on the current candidate mixture density. We use this smaller set of draws and weights to compute a new mode and scale matrix. The new mode and scale are used as starting parameters for the additional Student's $t$ component in the mixture. The reason for this choice is that the new Student's $t$ component should cover a part of the parameter space that is insufficiently covered by the previous candidate, when compared to the target density. The starting values for the mixture probability and degrees of freedom parameter for the new Student's $t$ component are set to 0.1 and 5 , respectively. The starting values of the mixture probabilities for the older Student's $t$ components are obtained by multiplying the latest values by 0.9. Given the last set of $N$ simulated draws and the corresponding importance weights, we apply the IS weighted EM algorithm to update the new mixture distribution. We simulate draws $\theta^{(1)}, \ldots, \theta^{(N)}$ from the updated proposal $q_{\zeta}$, and signal paths $x^{(1)}, \ldots, x^{(N)}$ conditionally on these parameter draws. We compute the corresponding IS weights $w^{(1)}, \ldots, w^{(N)}$ in (13).

(e) Evaluate the IS weights: We estimate the coefficient of variation (i.e., the standard deviation divided by the mean) of the IS weights of the last candidate. We terminate the iterations when the coefficient of variation changes by less than 5\%; otherwise we go to Step 1(d).

2. MCMC phase: We carry out the independent Metropolis-Hastings algorithm using the acceptance probability given in (5). 
In the training phase, there is a trade-off between the quality of the proposal and the speed of the estimation procedure. When more draws are used in the training phase, the approximation generally becomes better, but at the cost of an increased computation time. Fortunately, the draws and corresponding weights can be recycled such that we do not require the sampling of new draws when going through the iterations to obtain the mixture components. To be able to recycle previous draws, we need to implement a slight modification when computing the coefficient of variation of the importance weights that correspond to the latest candidate. Given the draws $\left(x^{(1)}, \theta^{(1)}\right), \ldots,\left(x^{(N)}, \theta^{(N)}\right)$ from the proposal $q_{0}(x, \theta \mid y)=q_{0}(x \mid \theta, y) q_{0}(\theta \mid y)$ with only one Student's $t$ component, we can evaluate the coefficient of variation of the weights based on the new proposal $q_{\zeta}(x, \theta \mid y)$ using the following results:

$$
\int \frac{p(x, \theta \mid y)}{q_{\zeta}(x, \theta \mid y)} q_{\zeta}(x, \theta \mid y) \mathrm{d} \theta \mathrm{d} x=\int \frac{p(x, \theta \mid y)}{q_{0}(x, \theta \mid y)} q_{0}(x, \theta \mid y) \mathrm{d} \theta \mathrm{d} x
$$

and

$$
\int \frac{p(x, \theta \mid y)^{2}}{q_{\zeta}(x, \theta \mid y)} \mathrm{d} \theta \mathrm{d} x=\int \frac{p(x, \theta \mid y)}{q_{0}(x \mid \theta, y) q_{\zeta}(\theta \mid y)} \frac{p(x, \theta \mid y)}{q_{0}(x, \theta \mid y)} q_{0}(x, \theta \mid y) \mathrm{d} \theta \mathrm{d} x .
$$

The variance of the importance weights corresponding to the latest candidate can then be estimated via

$$
\frac{1}{N} \sum_{j=1}^{N}\left[\frac{p\left(x^{(j)}, \theta^{(j)} \mid y\right)}{q_{0}\left(x^{(j)} \mid \theta^{(j)}, y\right) q_{\zeta}\left(\theta^{(j)} \mid y\right)} \frac{p\left(x^{(j)}, \theta^{(j)} \mid y\right)}{q_{0}\left(x^{(j)}, \theta^{(j)} \mid y\right)}\right]-\left[\frac{1}{N} \sum_{j=1}^{N} \frac{p\left(x^{(j)}, \theta^{(j)} \mid y\right)}{q_{0}\left(x^{(j)}, \theta^{(j)} \mid y\right)}\right]^{2}
$$

where $\left(x^{(j)}, \theta^{(j)}\right) \sim q_{0}(x, \theta \mid y)$ is an i.i.d. sequence for $j=1, \ldots, N$. This modification of the procedure leads to our modified JIMH method, which realizes a substantial gain in speed.

\subsection{Discussion and relation to other methods}

Since the most intensive part of the computations in our algorithm is the generation of the signal paths $x$ conditional on the parameters and observations, it is interesting to compare the number of signal draws that are required in our algorithm and in the PMCMC based methods. Our modified JIMH procedure requires $3 \times N+I$ draws where $N$ is the size of the training sample and $I$ is the number of iterations in the MCMC phase. In contrast, the PMCMC methods require $I \times S$ signal draws where $S$ is the number of draws used 
to estimate the likelihood. In the two examples in our simulation and empirical studies, we use $I=25,000, N=10,000$ and $S=50$. It implies that for the PMCMC we require around 20 times more signal paths than in our modified JIMH method.

Our estimation procedure and the method proposed by Pitt et al. (2012) are similar in spirit. The independent Metropolis-Hastings algorithm is the core of both algorithms. However, there are several clear differences between the two estimation routines. First, our method is not adaptive, which means that it is easier to paralellize as the proposal density is constant throughout the MCMC phase. Candidate draws are fully independent both in the training phase and in the MCMC phase. Second, we sample one state path at each iteration, instead of integrating out the state. This implies that we do not rely on the PMCMC arguments of Andrieu et al. (2010). We also emphasize that our method directly provides the smoothed state estimates; we do not require additional algorithms for this task. Finally, we use a mixture of Student's $t$-distributions instead of a mixture of normals to approximate the posterior distribution of the parameters.

The choice of the mixture of Student's $t$-densities as a proposal for the posterior of the parameters has several theoretical and practical advantages over other choices. First, under certain regularity conditions any density can be approximated by a mixture of Student's $t$-densities if we use a sufficient number of mixture components as shown by Zeevi and Meir (1997). Second, sampling from a mixture of Student's $t$-densities is fast. Third, the fat tails make the Student's $t$-distribution (with small enough degrees of freedom) a robust importance sampler. We are less prone to importance weights with an infinite variance and posterior estimates are more reliable and more efficient. Finally the construction of Student's t-distributions in the mixture and the mixing weights can be carried out efficiently by means of the MitISEM procedure, using the novel modification discussed in subsection 2.2 .

The exposition above concentrated on the case of a univariate signal. However, the method can be extended when the observation and signal are vectors by using the approach of Scharth (2012). This extended version of the NAIS method is able to treat the signal vector via the use of quasi-random numbers for the numerical evaluation of the variance of the log weights and subsequently for its minimization.

A promising feature of JIMH is that the evaluation of marginal likelihoods can take place in a straightforward way via importance sampling. On the basis of the proposal density obtained in the training phase we can approximate the marginal likelihood by

$$
p(y)=\int \frac{p(y \mid x ; \theta) p(x \mid \theta) p(\theta)}{q_{\zeta}(x, \theta \mid y)} q_{\zeta}(x, \theta \mid y) \mathrm{d} \theta \approx \frac{1}{N} \sum_{j=1}^{N} w^{(j)},
$$

where $w^{(j)}$ is defined as the right-hand side of (13), and $N$ is the number of draws used 
to evaluate the marginal likelihood.

The JIMH method does not provide a treatment for state space models with a nonlinear or a non-Gaussian state equation. However we emphasize here that the MitISEM approach remains feasible and valid when we use an unbiased estimator of the likelihood $\hat{p}\left(\theta^{(j)} \mid y\right)$ in equation (7) instead of the joint density $p\left(x^{(j)}, \theta^{(j)} \mid y\right)$ : both can be used to approximate the relevant term in the Kullback-Leibler divergence measure. Hence the MitISEM approach can still be applied as in Hoogerheide et al. (2012) with the only difference that we need to use the weights $\hat{w}^{(j)}=\hat{p}\left(\theta^{(j)} \mid y\right) / q_{0}\left(\theta^{(j)} \mid y\right)$ instead of the weights $p\left(\theta^{(j)} \mid y\right) / q_{0}\left(\theta^{(j)} \mid y\right)$ in $(7)$.

We are faced with a possible limitation of our method when the time dimension $T$ increases. In this case the variance of the importance weights also increases and the Monte Carlo approximation (11) may become unreliable. This can negatively affect the performance of both the MitISEM and JIMH procedures.

\section{Simulation study}

We carry out a detailed simulation experiment to demonstrate the performance of our estimation procedure against two alternative procedures. We estimate parameters for a stochastic volatility model and for a stochastic intensity model using simulated data sets. The stochastic volatility model is well known and provides an important benchmark model with many challenges for parameter estimation, see the discussions in Shephard (2005). Similar challenges emerge for the stochastic intensity model, but this model also illustrates a new and interesting application of a nonlinear non-Gaussian state space model for which the full conditional density of the parameter vector is not known in closed form. The stochastic intensity models are particularly used in portfolio credit risk modeling, see Koopman et al. (2008), Duffie et al. (2009) and Azizpour et al. (2010) for interesting illustrations of the problem. The details of the stochastic volatility and stochastic intensity models are given in Sections 3.2 and 3.3, respectively,

We choose to compare the performance of our proposed JIMH method with state-ofthe-art alternatives rather than with some feeble benchmark procedures. In particular, we compare the performances of parameter estimation by using the new JIMH method and by using two competing methods of Pitt et al. (2012). The first competing method is the adaptive random walk Metropolis-Hastings (ARWMH) algorithm and is an extension of the method of Roberts and Rosenthal (2009). Our second benchmark method is the adaptive independent Metropolis-Hastings (AIMH) algorithm where the proposal is a mixture of normals. It is an extension of the method of Giordani and Kohn (2010). 
These two recent and advanced methods provide fast and efficient solutions to parameter estimation for nonlinear non-Gaussian state space models by taking advantage of the powerful framework provided in Andrieu et al. (2010). To further enhance the numerical efficiency of the benchmark methodologies, we use a modified version of these methods by introducing the numerically accelerated importance sampling (NAIS) method of Koopman et al. (2011) for integrating out the signal vector. For the purpose of likelihood estimation, the NAIS method is used as an alternative to the partially adapted auxiliary particle filter of Pitt et al. (2012).

We use NAIS as a state sampler for the following three reasons: (i) it can provide an approximation to the state smoothing density that minimizes the variance of the log importance weights; (ii) the approximating Gaussian linear state space model can be constructed in a computationally efficient way by taking advantage of standard Kalman filter methods and deterministic integration methods for one-dimensional integrals; (iii) the simulated signal paths can be efficiently computed via the simulation smoothers of de Jong and Shephard (1995) or Durbin and Koopman (2002). We have found that the NAIS method yields estimates of the likelihood with lower variance and in less computing time than methods based on the particle filter. The findings are discussed in Section 3.1. In Appendix B we provide the details of the NAIS method. For its use in the ARWMH and AIMH methods, we use 50 simulated paths of the signal for likelihood estimation. We notice that JIMH requires one simulated signal path from NAIS only at each iteration. Further implementation details of the competitive benchmark methods are discussed in Appendices C and D.

We estimate the parameters for 56 data sets on an 8-core computer. The data sets are generated with parameter values that are close to those estimated from the empirical data sets of Section 4. For each simulated data set, we re-estimate the parameters using the JIMH method, its modified version (which we denote by JIMH mod.), the ARWMH method and the AIMH methods. For the modified JIMH method, the candidate draws are recycled after the first MitISEM update in the training phase. After 5,000 burn-in draws we perform 20,000 iterations of the algorithms. We calculate medians and interquartile ranges (over the 56 simulated data sets) of the parameter estimates, acceptance rates, and inefficiencies. In order to assess the quality of the simulation methods we compute the inefficiency factor $(I F)$, which is defined as the variance of the parameter estimate divided by the variance in case the sampling scheme would generate independent posterior draws. The $I F$ statistic is discussed, amongst others, by Pitt et al. (2012). In our case, 
we define the inefficiency factor as

$$
I F=1+2 \sum_{j=1}^{\max (L, 1000)} r_{j}
$$

where $r_{j}$ is the $j$-th order sample serial correlation amongst the 20,000 parameter draws, and where $L$ is the lowest order $j$ for which $r_{j}$ is not significant.

\subsection{Likelihood estimation: NAIS versus particle filters}

We have discussed that the ARWMH and AIMH methods can be based on both the PMCMC and NAIS algorithms for drawing the signal vectors. To assess the difference between the two implementations of the methods, we use the particle filter and NAIS methods to evaluate the likelihood function. A review of different particle filtering methods is provided in Doucet et al. (2001). For the case of a stochastic volatility model, we obtain more efficient likelihood estimates when using NAIS in comparison to using particle filters. The NAIS importance sampling estimates of the likelihood function have lower variance and need less computing time than the particle filter likelihood estimates.

We simulate 56 data sets using the same data generation process for the stochastic volatility model of Section 3.2. We estimate the likelihood value at the "true" parameter values 100 times for each simulated data set using the bootstrap filter of Gordon et al. (1993), the auxiliary particle filter of Pitt and Shephard (1999) and the NAIS method of Koopman et al. (2011). We compute 100 likelihood estimates for each data set and we calculate the variance of the estimates together with the mean computing time for each data set. We report the median variance and computing times over the 56 data sets. Table 1 presents the results. For all considered time series lengths, the median variance of the NAIS estimate is lower than the median variance of the particle filter estimates for all numbers of particles considered. Moreover, the estimation using importance sampling takes much less time than the estimation using particle filters. We therefore use the NAIS in all algorithms to create a level playing field. 
Table 1: Comparison of the variance of the particle filter and importance sampling estimate of the likelihood in the SV model based on 56 simulated data sets. We compare the likelihood estimates from the bootstrap filter (BF), the auxiliary particle filter (APF) and the numerically accelerated importance sampling (NAIS). $M$ denotes the number of particles/number of draws. $T$ is the length of the simulated data set. For different $M$ and $T$ we report the median of the variances over the 56 data sets, where these variances are calculated from 100 runs per data set.

\begin{tabular}{lrrrrr}
\hline \multirow{2}{*}{ Method } & \multirow{2}{*}{$\mathrm{M}$} & \multicolumn{2}{c}{ Variance } & \multicolumn{2}{c}{ Time } \\
\cline { 3 - 6 } & & $\mathrm{T}=1000$ & $\mathrm{~T}=2000$ & $\mathrm{~T}=1000$ & $\mathrm{~T}=2000$ \\
\hline \multirow{3}{*}{$\mathrm{BF}$} & 250 & 1.079 & 1.505 & 5.970 & 37.901 \\
& 500 & 0.743 & 1.071 & 18.435 & 88.828 \\
& 1000 & 0.541 & 0.735 & 44.018 & 173.432 \\
\hline \multirow{3}{*}{$\mathrm{APF}$} & 250 & 0.988 & 1.420 & 6.362 & 37.434 \\
& 500 & 0.674 & 0.963 & 21.025 & 88.764 \\
& 1000 & 0.490 & 0.687 & 42.663 & 195.456 \\
\hline \multirow{2}{*}{ NAIS } & 50 & 0.140 & 0.233 & 0.283 & 0.527 \\
\hline
\end{tabular}

\subsection{Stochastic volatility model}

Many macroeconomic and financial time series exhibit volatility clustering, which results in autocorrelated time varying variances and volatilities. To capture autocorrelation in volatilities we can adopt the stochastic volatility (SV) model. A basic specification of the stochastic volatility model is given by

$$
\begin{aligned}
y_{t} & =\exp \left(x_{t} / 2\right) \varepsilon_{t}, & & \varepsilon_{t} \sim \mathrm{N}(0,1), \\
x_{t}-\delta & =\phi\left(x_{t-1}-\delta\right)+\eta_{t}, & & \eta_{t} \sim \mathrm{N}\left(0, \sigma_{\eta}^{2}\right),
\end{aligned}
$$

where $x_{t}$ is the unobserved log-volatility process, which follows an autoregressive process of order $1, \varepsilon_{t}$ is a standardized error term, $\delta$ is the overall mean of $x_{t}, 0<\phi<1$ is the persistence parameter, and $\sigma_{\eta}^{2}>0$ is the innovation variance of the log-volatility process. The three unknown parameters $\delta, \phi$, and $\sigma_{\eta}^{2}$ need to be estimated. More discussions on the SV model and its extensions can be found in Kim et al. (1998) and Shephard (2005).

The 56 data sets are generated from the basic SV model (20) and (21) with parameters set equal to $\delta=0.48, \phi=0.97$, and $\sigma_{\eta}^{2}=0.049$, which correspond closely to the estimates obtained in our empirical study in Section 4. We simulate time series of length 1,250 and use the following prior specifications for the parameters

$$
\delta \sim \mathrm{N}(0,1), \quad \frac{\phi+1}{2} \sim \operatorname{Beta}(20,1.5), \quad \frac{1}{\sigma_{\eta}^{2}} \sim \operatorname{Gamma}\left(\frac{5}{2}, \frac{0.05}{2}\right) .
$$

Table 2 presents the medians and interquartile ranges of acceptance rates, parameter estimates and inefficiency factors. We also present the estimated posterior means of the 
Table 2: Performance for the Stochastic Volatility model: acceptance rates, parameter estimates, and inefficiency factors (19) for different Metropolis-Hastings algorithms. The table presents the medians and interquartile ranges (within parentheses) over 56 simulated data sets. The estimates are based on 20,000 draws after a burn-in sample of 5,000 observations. We use 50 simulated draws to evaluate the likelihood in the ARWMH and AIMH algorithms.

\begin{tabular}{lcccccccc}
\hline \multirow{2}{*}{ Algorithm } & \multirow{2}{*}{ Time (in s) } & \multirow{2}{*}{ Ac. Rate } & \multicolumn{3}{c}{ Estimate } & \multicolumn{3}{c}{ Inefficiency } \\
\cline { 5 - 9 } & & & $\delta$ & $\phi$ & $\sigma_{\eta}^{2}$ & $\delta$ & $\phi$ & $\sigma_{\eta}^{2}$ \\
\hline ARWMH & 8401 & 0.305 & 0.445 & 0.971 & 0.049 & 14.538 & 14.312 & 13.265 \\
\multirow{2}{*}{ AIMH } & $(485)$ & $(0.017)$ & $(0.195)$ & $(0.014)$ & $(0.016)$ & $(2.588)$ & $(2.151)$ & $(2.079)$ \\
& 8020 & 0.604 & 0.445 & 0.971 & 0.049 & 2.905 & 2.979 & 2.900 \\
JIMH 10k & $(277)$ & $(0.092)$ & $(0.204)$ & $(0.014)$ & $(0.016)$ & $(0.687)$ & $(1.719)$ & $(1.390)$ \\
& 3526 & 0.507 & 0.450 & 0.970 & 0.048 & 4.833 & 5.070 & 5.561 \\
JIMH mod.10k & 2406 & 0.509 & 0.442 & 0.971 & 0.049 & 5.113 & 5.056 & 5.572 \\
& $(1062)$ & $(0.065)$ & $(0.195)$ & $(0.014)$ & $(0.016)$ & $(1.106)$ & $(2.139)$ & $(2.210)$ \\
JIMH mod. 2k & 1417 & 0.501 & 0.444 & 0.971 & 0.049 & 6.354 & 5.471 & 4.970 \\
& $(75)$ & $(0.061)$ & $(0.201)$ & $(0.013)$ & $(0.017)$ & $(3.398)$ & $(3.324)$ & $(2.818)$ \\
\hline
\end{tabular}

parameters to indicate that the different simulation methods provide similar results.

The results suggest that none of the methods considered produce biased estimates due to the omission of relevant parts of the parameter space. Therefore we focus the comparisons of the alternative methods in terms of their differences in accuracy and computing time.

The interquartile ranges of the estimated posterior means are similar among the different methods. This finding does not imply that for example ARWMH is performing as well as the other methods, because the interquartile ranges are mainly driven by the differences between the 56 simulated data sets and in particular by the differences between the 56 true posterior means. The reported inefficiency factors $(I F)$ in the last three columns of the table suggest that the ARWMH method is close to 3-4 times less efficient than the competing methods. The medians of the inefficiency factor and the acceptance rates show that the AIMH is successful in approximating the posterior density of the parameters. The interquartile range of the inefficiency factors are indicative of the robustness of the methods across different data sets. The JIMH methods perform slightly worse compared to the AIMH approach for both acceptance rates and inefficiencies. However, the JIMH methods requires substantially less computing time. The modified version of JIMH provides a slightly lower quality proposal than the standard JIMH, but comes 
Figure 2: Performance for the Stochastic Volatility model: average effective sample size per computing time (in seconds). The average (over the three parameters) of the $\operatorname{ESS}(s)$ is computed for one simulated data set for the different estimation procedures. The average $E S S(s)$ is calculated every five minutes, after which linear interpolation gives a crude approximation of the average $E S S(s)$ as a function of computing time.

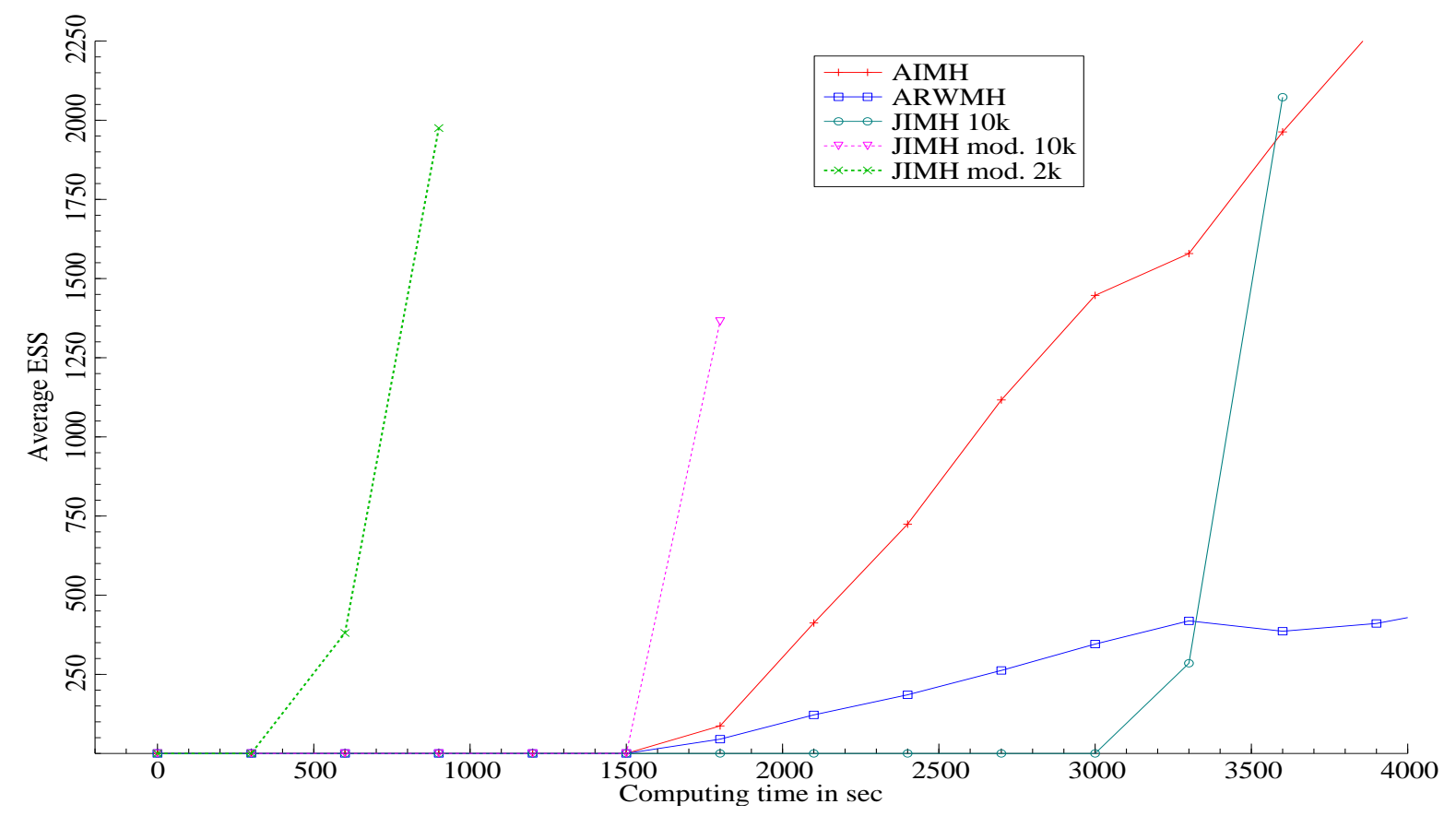

with a clear reduction in computing time. Finally, the results for modified JIMH with a training sample of only 2,000 draws (instead of the default of 10,000 draws) suggest that we are able to obtain further efficiency gains at the cost of a moderate loss of robustness of the procedure.

To obtain further insight in the efficiency of the estimation procedures, we look at the trade-off between the inefficiency factor and computing time. We obtain a crude approximation of the effective sample size as a function of computing time. During the estimation process, after each five-minute period, we approximate the effective sample size by $E S S(s)=N(s) / I F(s)$, where $N(s)$ and $I F(s)$ are the number of draws (after the discarded burn-in sample) and the inefficiency factor in period $s$, respectively. We report the average $E S S(s)$ for the three parameters for a randomly chosen simulated data set in Figure 2. Similar patterns are obtained for the other simulated data sets.

For the AIMH and ARWMH algorithms it takes five periods of five minutes (1500 seconds) to draw the burn-in sample. After 1500 seconds, the average $E S S(s)$ value starts to increase. The average $E S S(s)$ for AIMH increases more steeply than for ARWMH since the inefficiencies are higher for ARWMH, while the computing times are similar because both methods integrate out the state vector at each iteration. The $E S S(s)$ slopes for 
JIMH are much steeper than for AIMH and ARWMH. This confirms the efficiency and good acceptance rates of JIMH. A smaller size of the training sample or the use of the modified version of the JIMH algorithm clearly lead to further efficiency gains.

\subsection{Stochastic intensity model}

For our second simulation experiment, we consider a stylized version of the point processes model with stochastic intensity as used in our second empirical application in Section 4.2. Koopman et al. (2008) and Duffie et al. (2009) consider the stochastic intensity model for studying the systematic dynamics of U.S. corporate defaults and credit rating migrations.

For the simulation version of the model, we consider a pool of $K$ firms and a jump process $y_{k}(t)$ for each firm $k=1, \ldots, K$ with common jump intensity $\lambda(t)$ as given by

$$
\lambda(t)=\exp \left[\omega+\beta^{\prime} c(t)+\gamma x(t)\right]
$$

where $\omega$ is the base log-intensity, $\beta$ is a vector of regression parameters, $c(t)$ is a vector of covariates and $\gamma$ is a scale factor for the unobserved signal $x(t)$. The cumulative jump process over all firms is given by

$$
y(t)=\sum_{k=1}^{K} y_{k}(t)
$$

The signal $x(t)$ is often referred to as an unobserved frailty factor. We follow standard practice and model it as a zero mean Ornstein-Uhlenbeck (OU) process, standardized to have unit variance at $t=1$,

$$
\mathrm{d} x(t)=-\rho x(t) \mathrm{d} t+\sqrt{2 \rho} \mathrm{d} W(t)
$$

where $\rho>0$ is a persistence parameter and $W(t)$ is a standard Brownian motion. The set of covariates we use in the simulation is the same as in the empirical section, namely the (i) one year difference of the S\&P500 index, (ii) term spread between the 10-year and 1-year Treasury Bond (with constant maturity rates), (iii) secondary market rate on 3 month Treasury Bills, and (iv) year-to-year percentage change of US industrial production (final output), all at the monthly frequency over the period from January 1, 1970 to March 4, 2010; compare Duffie et al. (2007), Lando and Nielsen (2010) and Azizpour et al. (2010). The covariates are obtained from the FRED and CRSP databases. We set the parameters to $\omega=-4.75, \beta=(-0.85,0.01,-0.055,-5.1), \gamma=1.15$ and $\rho=0.12$, which are close to the empirical estimates from Section 4. 
We simulate data for $K=3,000$ firms over the period January 1, 1970 to March 4, 2010. As $K$ is kept fixed, a firm can jump repeatedly over the sample. If a jump is interpreted as default, this implies that the firm is directly re-started after default at the same pre-default intensity. In the empirical application, we depart from this construction and allow for an absorbing default state as well as for firms that enter the sample or leave the sample for other reasons than default.

The simulations are conditional on the four covariates and are sampled by using a discretization of the continuous time processes $y(t), x(t)$ and $\lambda(t)$, where the discretization takes steps of $1 / 32$ part of a day. Within each $1 / 32$ part of the day, we use a Bernoulli approximation to generate defaults. We generate 56 data sets in this way. In starting the estimation process, we use (weakly informative) uniform priors on relatively wide intervals: $[-8,-2]$ for $\omega,[0.01,3]$ for $\gamma,[0.01,1]$ for $\rho$ and $[-20,20]$ for each of the four elements of $\beta$.

We consider the $i$ th event time $t_{i}$ and define the indicator variable $D_{k i}$ to be one, $D_{k i}=1$, if firm $k$ jumps at the $i$ th event time $t_{i}$, and zero otherwise. The number of jumps at event time $t_{i}$ over all firms is given by $D_{i}=\sum_{k=1}^{K} D_{k i}$. The discrete time approximation of the jump process $y(t)$ leads to the following dynamic model in event time,

$$
\begin{array}{rlrl}
p\left(y_{i} \mid x_{i}, \theta\right) & =\exp \left[D_{i} \log \lambda_{i}-\lambda_{i} K \Delta_{i}\right], & \\
x_{i} & =e^{-\rho \Delta_{i}} x_{i-1}+\eta_{i}, \quad \eta_{i} \sim \mathrm{N}\left(0,1-e^{-2 \rho \Delta_{i}}\right),
\end{array}
$$

where $p\left(y_{i} \mid x_{i}, \theta\right)$ is the density of $y_{i}=y\left(t_{i}\right)$ conditional on signal $x_{i}=x\left(t_{i}\right)$ and parameter vector $\theta$, with $\lambda_{i}=\lambda\left(t_{i}\right)$ and $\Delta_{i}=t_{i}-t_{i-1}$. Further details of the model are presented at the empirical application in Section 4.2.

Table 3 presents the means and interquartile ranges of the parameter estimates for the 56 simulated data sets. The different simulation methods provide similar results, which suggests that none of the methods provides biased estimates. Table 4 presents the means and interquartile ranges of acceptance rates and inefficiency factors. We find that the ARWMH algorithm is clearly outperformed by the other methods. Moreover, for the stochastic intensity model, the AIMH algorithm performs generally less favourable compared to JIMH. Further, the AIMH method appears to be less robust for certain simulated data sets. The median inefficiencies are higher and also the interquartile ranges of the inefficiencies are larger compared to JIMH. A possible explanation is that AIMH uses a mixture of normal distributions, such that the algorithm can sometimes fail at parameter draws from the tails. In contrast, the JIMH method uses a mixture of Student's $t$-densities to approximate the posterior distribution of the parameters. The fat tails of 
Table 3: Performance for the stochastic intensity model: parameter estimates for different Metropolis-Hastings algorithms. The table presents the medians and interquartile ranges (within parentheses) over 56 simulated data sets. The estimates are based on 20,000 draws after a burn-in sample of 5,000 observations. We use 50 simulated draws to evaluate the likelihood in the ARWMH and AIMH algorithms.

\begin{tabular}{lccccccc}
\hline & \multicolumn{7}{c}{ Estimate } \\
\cline { 2 - 8 } & $\omega$ & $\beta_{1}$ & $\beta_{2}$ & $\beta_{3}$ & $\beta_{4}$ & $\gamma$ & $\rho$ \\
\hline True values & -4.75 & -0.85 & 0.01 & -0.055 & -5.1 & 1.15 & 0.12 \\
Algorithm: & & & & & & & \\
ARWMH & -4.559 & -0.909 & 0.021 & -0.044 & -4.611 & 1.307 & 0.154 \\
& $(1.212)$ & $(0.498)$ & $(0.202)$ & $(0.107)$ & $(2.280)$ & $(0.400)$ & $(0.111)$ \\
AIMH & -4.621 & -0.895 & 0.026 & -0.043 & -4.546 & 1.256 & 0.149 \\
& $(1.247)$ & $(0.501)$ & $(0.206)$ & $(0.103)$ & $(2.330)$ & $(0.459)$ & $(0.111)$ \\
JIMH 10k & -4.624 & -0.896 & 0.025 & -0.044 & -4.591 & 1.286 & 0.151 \\
& $(1.183)$ & $(0.483)$ & $(0.209)$ & $(0.108)$ & $(2.182)$ & $(0.382)$ & $(0.106)$ \\
JIMH mod.10k & -4.580 & -0.899 & 0.024 & -0.044 & -4.562 & 1.283 & 0.150 \\
& $(1.220)$ & $(0.482)$ & $(0.211)$ & $(0.105)$ & $(2.219)$ & $(0.389)$ & $(0.107)$ \\
JIMH mod. 5k & -4.599 & -0.897 & 0.023 & -0.044 & -4.559 & 1.302 & 0.145 \\
& $(1.189)$ & $(0.477)$ & $(0.206)$ & $(0.106)$ & $(2.115)$ & $(0.394)$ & $(0.106)$ \\
\hline
\end{tabular}

the Student's $t$-density prevent that the MH method repeats a draw from one of the tails for a long sequence of iterations. The performance of the modified versions of JIMH are again comparable to the standard version. According to the results in Table 4, the size of the training sample can be reduced to obtain higher efficiency gains.

Figure 3 presents the average $E S S(s)$ per computing time for the different methods and for a randomly chosen data set. We can see that the relative performance of the alternative methods for the stochastic intensity model is similar to that for the stochastic volatility model. After the burn-in draws are computed in the first 3,000 seconds, the average $E S S(s)$ for the AIMH and ARWMH methods starts to increase relatively slowly compared to that of JIMH. It shows that the JIMH methods are computationally more efficient. They outperform the alternative methods both in terms of computing speed and in terms of the fit of the proposal. Furthermore, Figure 3 shows for this particular data set that the average $E S S(s)$ of AIMH is only increasing steadily after 6300 seconds. The decrease and standstill of the estimated average $E S S(s)$ between 5100 and 6300 seconds is caused by having a long sequence of draws at a particular parameter value in a remote part of the posterior distribution that had not yet been explored by earlier draws (that were simulated in the first 5100 seconds). 
Table 4: Performance for the stochastic intensity model: acceptance rates and inefficiency factors (19) for different Metropolis-Hastings algorithms. The table presents the medians and interquartile ranges (within parentheses) over 56 simulated data sets. The estimates are based on 20,000 draws after a burn-in sample of 5,000 observations. We use 50 simulated draws to evaluate the likelihood in the ARWMH and AIMH algorithms.

\begin{tabular}{lccccccccc}
\hline \multirow{2}{*}{ Algorithm } & Time & Acc. & \multicolumn{7}{c}{ Inefficiency } \\
\cline { 6 - 11 } & (in s) & Rate & $\omega$ & $\beta_{1}$ & $\beta_{2}$ & $\beta_{3}$ & $\beta_{4}$ & $\gamma$ & $\rho$ \\
\hline ARWMH & 10053 & 0.247 & 41.833 & 31.735 & 29.212 & 29.428 & 32.037 & 39.873 & 33.523 \\
& $(7380)$ & $(0.015)$ & $(13.621)$ & $(6.883)$ & $(4.619)$ & $(5.650)$ & $(7.931)$ & $(21.481)$ & $(1.469)$ \\
AIMH & 8953 & 0.411 & 25.256 & 8.308 & 10.812 & 9.439 & 8.592 & 26.513 & 10.777 \\
\multirow{2}{*}{ JIMH 10k } & 5858 & 0.658 & 3.431 & 2.586 & 2.575 & 2.624 & 2.642 & 3.406 & 2.893 \\
& $(3684)$ & $(0.031)$ & $(0.769)$ & $(0.374)$ & $(0.380)$ & $(0.327)$ & $(0.559)$ & $(0.879)$ & $(0.666)$ \\
JIMH mod.10k & 3124 & 0.633 & 4.863 & 2.900 & 2.884 & 2.899 & 3.024 & 4.934 & 3.355 \\
& $(2386)$ & $(0.041)$ & $(3.950)$ & $(0.735)$ & $(0.804)$ & $(0.946)$ & $(0.728)$ & $(5.605)$ & $(1.210)$ \\
JIMH mod. 5k & 2089 & 0.618 & 5.877 & 3.265 & 3.169 & 3.279 & 3.303 & 5.926 & 4.117 \\
& $(1616)$ & $(0.037)$ & $(4.823)$ & $(0.851)$ & $(1.012)$ & $(0.985)$ & $(1.062)$ & $(7.377)$ & $(2.038)$ \\
\hline
\end{tabular}

Figure 3: Performance for the stochastic intensity model: average effective sample size per computing time (in seconds). The average (over the seven parameters) of the $E S S(s)$ is computed for one simulated data set for the different estimation procedures. The average $E S S(s)$ is calculated every five minutes, after which linear interpolation gives a crude approximation of the average $E S S(s)$ as a function of computing time.

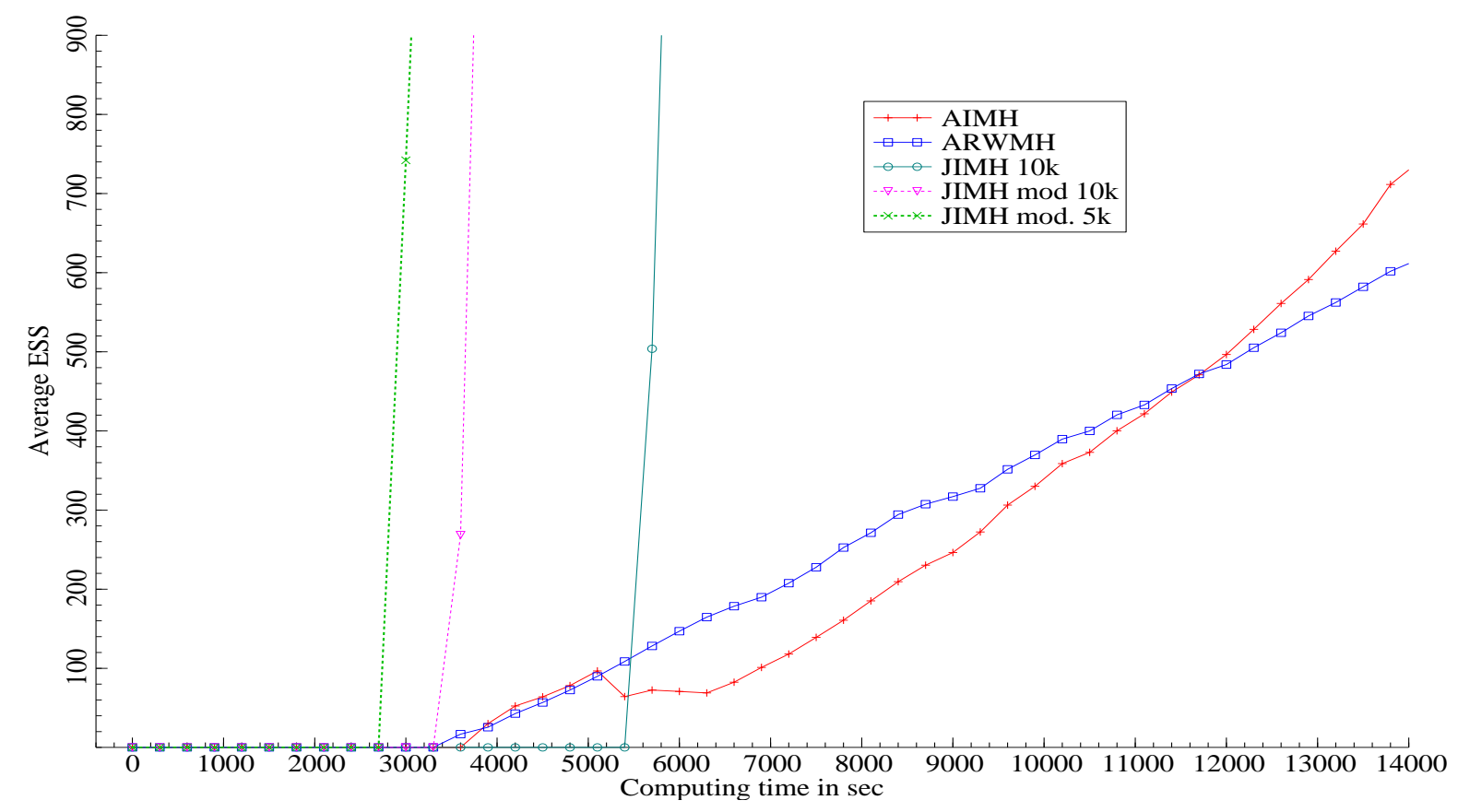


We conclude from the results presented for both stochastic volatility and stochastic intensity models that the JIMH algorithm offers an efficient alternative to PMCMC. Substantial improvements are obtained with respect to computational speed, accuracy, numerical efficiency, and robustness.

\section{Empirical examples}

In this section we illustrate the performance of the Joint Independent Metropolis-Hastings method using empirical data. First, we estimate the parameters and the signal for the stochastic volatility model for a time series of daily IBM stock returns. Second, we estimate the stochastic intensity model using a large panel of U.S. corporate defaults.

\subsection{Stochastic volatility model}

We consider the stochastic volatility model for IBM stock returns over the period January 3, 2007 to December 30, 2011. The data are obtained from CRSP. The sample period includes the financial crisis at the end of 2008 and the subsequent recession. Table 5 presents a selection of descriptive statistics. The average return is close to zero. The excess kurtosis and skewness indicate that the density of returns is heavy-tailed and nearly symmetrically distributed. The sample autocorrelation functions of the returns and squared returns are shown in Figure 4. The autocorrelations of the squared returns clearly display that the returns exhibit volatility clustering.

The estimated parameters and the inefficiencies of the chains of JIMH draws are reported in Table 6 . The estimated parameter values are typical of what is found in similar empirical studies. The unconditional mean of the log volatility process is estimated close to 0.48 . The estimated autoregressive coefficient in the state equation is 0.97 , which implies a highly persistent log-volatility process.

The inefficiency of the posterior draws is 5 for the mean $\delta$ and persistence coefficient $\phi$, and 10 for the variance $\sigma_{\eta}^{2}$. The acceptance rate for JIMH is $50.85 \%$. Figure 6 displays the IBM return series along with the smoothed signal estimates. The JIMH method provides these smoothed estimates and the corresponding confidence band as a direct byproduct of the algorithm: they are computed directly from the accepted MH draws of the signal paths. We observe increased volatility levels at the end of 2008, the first half of 2009, and at the end of 2011.

As mentioned earlier, the computation of marginal likelihoods is also straightforward 
Table 5: Descriptive statistics for the IBM returns from January 3, 2007 to December 30, 2011.

\begin{tabular}{lc}
\hline & IBM \\
\hline Number of obs. & 1260 \\
Mean & 0.051 \\
Standard deviation & 1.621 \\
Skewness & 0.095 \\
Kurtosis & 7.004 \\
\hline
\end{tabular}

Table 6: Stochastic Volatility model: parameter estimates for IBM returns from January 3, 2007 to December 30, 2011. The $90 \%$ credible intervals are within parentheses. The inefficiency factor is computed as (19).

\begin{tabular}{lcc}
\hline Parameter & Estimate & Inefficiency \\
\hline$\delta$ & 0.479 & 4.979 \\
& $(0.065,0.875)$ & \\
$\phi$ & 0.973 & 5.849 \\
& $(0.956,0.988)$ & \\
$\sigma_{\eta}^{2}$ & 0.049 & 9.639 \\
\hline
\end{tabular}

in the JIMH approach. We obtain the marginal likelihood estimate as the average of the importance sampling weights. These weights have already been computed during the algorithm to construct the $\mathrm{MH}$ acceptance probabilities. As an alternative to the independent Metropolis-Hastings method, we could use importance sampling to estimate the model parameters or smoothed log volatility process $x(t)$ using the candidate draws and corresponding importance weights obtained during the JIMH algorithm. As the candidate draws are independent, we would not have to discard burn-in draws. Also, the independence allows us to more easily compute reliable numerical standard errors for the estimated posterior means. However, when using importance sampling the computation of the posterior density of a parameter or of its confidence band would require additional works. This stems from the fact that the importance sampling method yields a series of weighted candidate draws instead of draws from the posterior itself. In any case, the results from importance sampling and from the independent $\mathrm{MH}$ algorithm are typically rather close if based on the same set of draws. 
Figure 4: Sample autocorrelation functions for the IBM returns (top panel) and for the squared IBM returns (bottom panel) from January 3, 2007 to December 30, 2011.
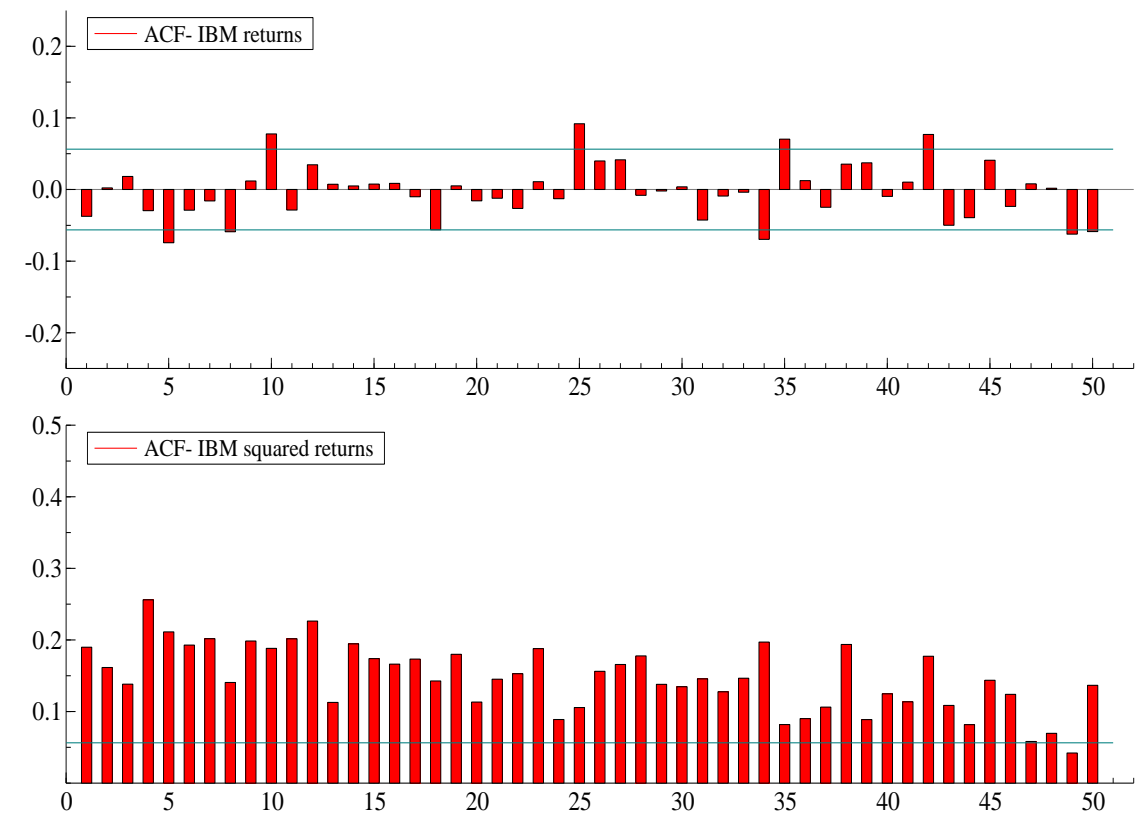

Figure 5: IBM returns from January 3, 2007 to December 30, 2011 (top panel) and the smoothed estimate of the log volatility process with its $90 \%$ credible interval (bottom panel).

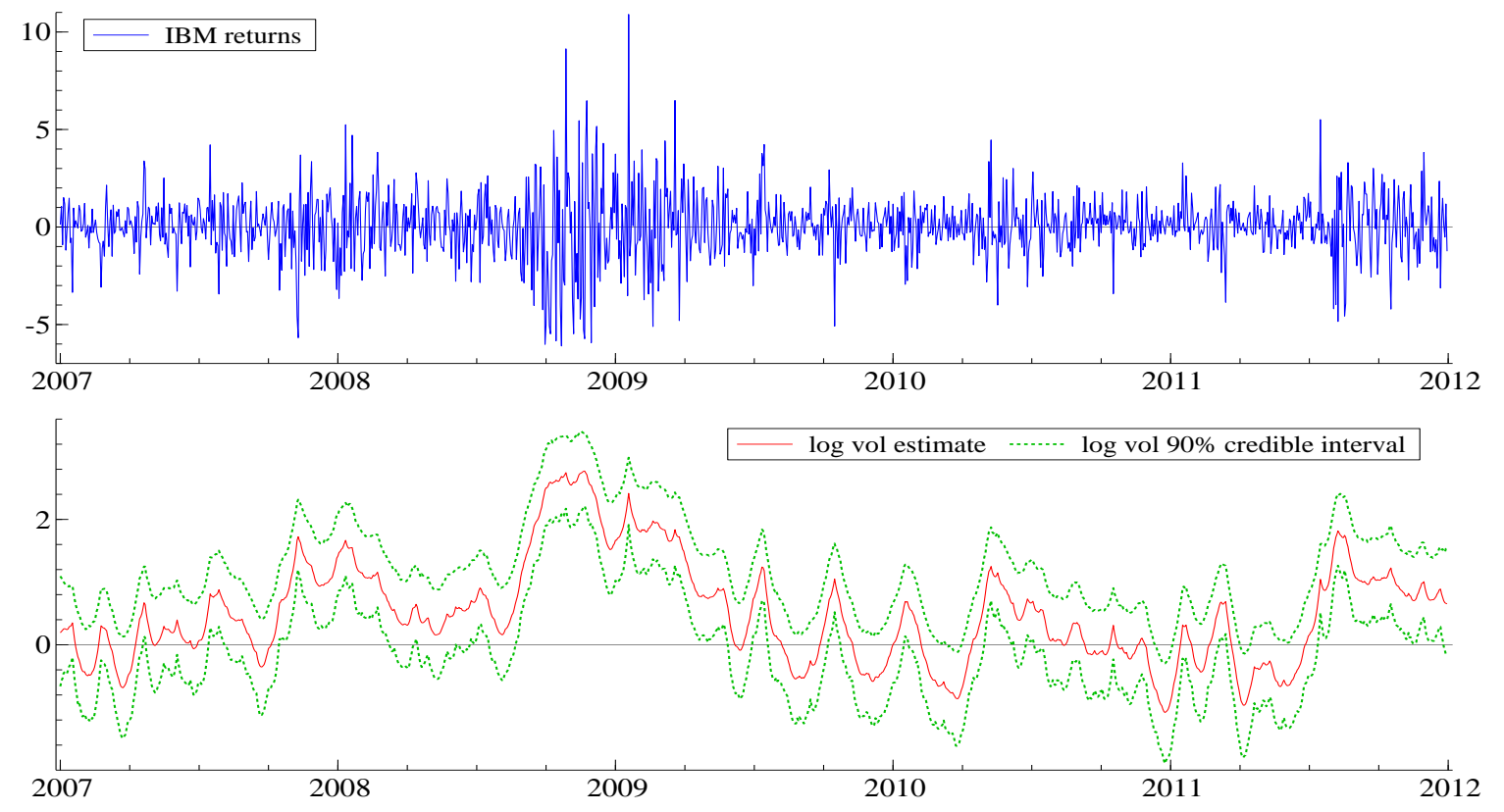




\subsection{Stochastic intensity model}

For our second empirical illustration we consider the stochastic intensity model for a large panel data set of U.S. corporate defaults obtained from Moody's. The core of the model is the same as in Section 3.3, with a slight change to account for sample extension and attrition for other reasons than default. The dummy variable $D_{k i}$ is defined as before, with $D_{k i}=1$ if firm $k$ jumps into default at time $t_{i}$, and $D_{k i}=0$ otherwise. We introduce the new dummy variables $R_{k i}$, with $R_{k i}=1$ if firm $k$ is at risk of defaulting at time $t_{i}-\varepsilon$, for $\varepsilon>0$ arbitrarily small, and $R_{k i}=0$ otherwise. An event time $t_{i}$ occurs when one of the control variates changes its value (e.g., at the end of the month or quarter), when a firm is added to the sample, or when a firm leaves the sample, either due to default or due to other reasons. We denote the default intensity of firm $k$ at time $t_{i}$ as $\lambda_{k i}=\lambda_{k}\left(t_{i}\right)$.

The conditional density of the observations given the complete paths of the covariates $c_{i}=c\left(t_{i}\right), i=1, \ldots, T$ and the complete path of the unobserved process $x_{i}=x\left(t_{i}\right), i=$ $1, \ldots, T$ is given by

$$
p(y \mid x, \theta)=\prod_{i=1}^{T} p\left(y_{i} \mid x_{i}, \theta\right)=\prod_{i=1}^{T} \prod_{k=1}^{K} \exp \left[D_{k i} \log \lambda_{k i}-R_{k i} \lambda_{k i} \Delta_{i}\right],
$$

for $y=\left(y_{1}, \ldots, y_{T}\right)^{\prime}$ and $x=\left(x_{1}^{\prime}, \ldots, x_{T}^{\prime}\right)^{\prime}$. Firm $k$ only contributes to the likelihood function when it is at risk of defaulting, that is when $R_{k i}=R_{k}\left(t_{i}\right)=1$. The state equation remains the same as in Section 3.3.

Our data set contains 1,627 defaults from 12,881 U.S. firms observed daily over the period January 1, 1970 to March 4, 2010. The number of firms in the portfolio increases over time from about 1,000 firms at the beginning of the sample to around 5,000 firms around 2010. Defaults originating from parent-subsidiary relationships are excluded: if there are multiple defaults with the same parental ID, we only keep the oldest firm as this is likely to be the parent firm. Event times and durations are measured in business days. As in Koopman et al. (2008), we winsorize the number of defaults per day to one to account for outliers and other data irregularities, see Koopman et al. (2008) for further details on data cleaning.

The top panel of Figure 6 shows the number of defaults per day. The concentration of the vertical lines clearly presents evidence of default clustering over time. In particular, we find high levels of defaults during 1989-1991 at the end of the savings and loan crisis and during the subsequent recession, in 2001 after the burst of the dot-com bubble, and after the 2008 financial crisis and during the subsequent recession. The increasing numbers of defaults in the last two decades do not immediately imply an increase in the frailty process $x(t)$, because also the number of firms increased substantially in the later part of 
Table 7: Stochastic intensity model: parameter estimates for U.S. corporate defaults from January 1, 1970 to March 4, 2010. The $90 \%$ credible intervals are within parentheses. The inefficiency factor is calculated as (19).

\begin{tabular}{|c|c|c|c|}
\hline & Parameter & Estimate & Inefficiency \\
\hline$\omega$ & Constant & $\begin{array}{c}-4.750 \\
(-6.357,-3.287)\end{array}$ & 2.755 \\
\hline$\beta_{1}$ & S\&P500 1 year return & $\begin{array}{c}-0.862 \\
(-1.346,-0.375)\end{array}$ & 2.097 \\
\hline$\beta_{2}$ & Term spread & $\begin{array}{c}0.013 \\
(-0.164,0.191)\end{array}$ & 3.495 \\
\hline$\beta_{3}$ & 3M TBill & $\begin{array}{c}-0.054 \\
(-0.167,0.058)\end{array}$ & 2.489 \\
\hline$\beta_{4}$ & Change in indust. prod. & $\begin{array}{c}-5.115 \\
(-7.798,-2.292)\end{array}$ & 2.450 \\
\hline$\gamma$ & Loading on frailty & $\begin{array}{c}1.148 \\
(0.548,2.294)\end{array}$ & 2.809 \\
\hline$\rho$ & Mean reversion & $\begin{array}{c}0.120 \\
(0.014,0.351)\end{array}$ & 2.688 \\
\hline
\end{tabular}

the sample. Furthermore, the covariates may also partly explain the movements in the data. The covariates are S\&P500 returns, Treasury Bond spreads, 3 month Treasury Bill yields, and yearly changes in U.S. industrial production, see also Section 3.3.

The parameter estimates and the inefficiency factors for the chains of the JIMH draws are presented in Table 7 . The $90 \%$ credibility intervals of the coefficients $\beta_{2}$ (for the term spread) and $\beta_{3}$ (for the U.S. Treasury Bill rate) include zero which indicates that these parameters are not significantly different from zero. The signs of the parameters are consistent with what we expect. Both lower returns on the S\&P500 index and lower percentage changes in industrial production imply a higher default intensity. The mean reversion parameter $\rho$ is estimated as 0.12 . At the yearly frequency, this implies an autoregressive coefficient $e^{-\rho} \approx 0.9$, such that the frailty process has a high persistence. The inefficiency factors have values around 3 and the acceptance rate is $71.04 \%$. The bottom panel of Figure 6 displays the smoothed estimate of the frailty process together with the $90 \%$ confidence interval. We emphasize that the confidence interval includes all uncertainties due to the observation noise, the randomness of the frailty process, and the uncertainty about the parameter vector $\theta$. This contrasts with the confidence bands around the estimated frailty process as based on classical analysis and as shown in most 
Figure 6: Default data from 1st January 1970 to 4th March 2010 (top panel) and the smoothed estimate of the frailty process with its $90 \%$ credible interval (bottom panel).

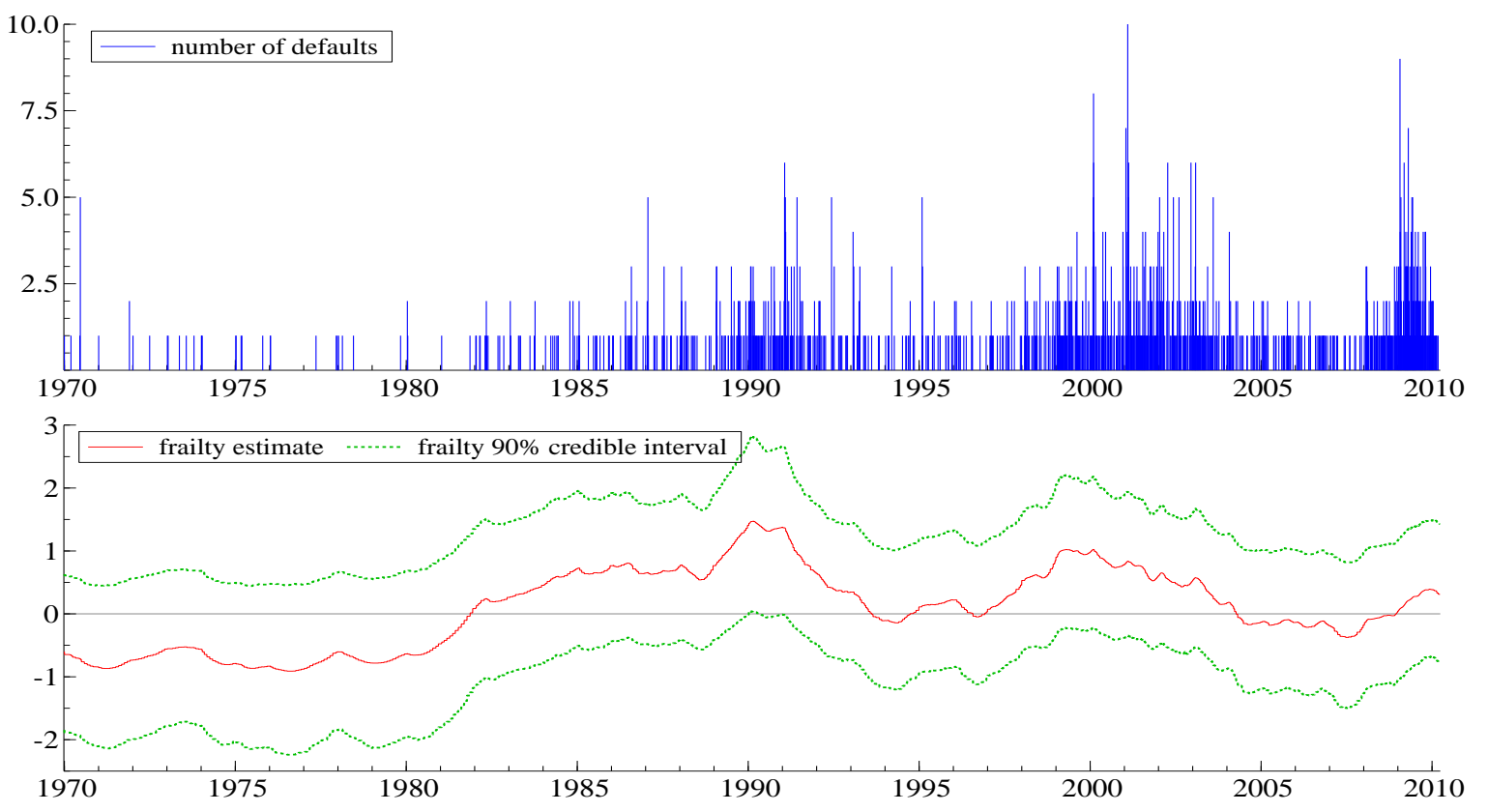

of the literature. There, the parameter uncertainty is usually ignored. The estimated frailty process represents the credit cycle dynamics in excess of the dynamics caused by the observable controls in $c\left(t_{i}\right)$. We clearly recognize the local peaks of the 1991 recession, the burst of the dot-com bubble, and the aftermath of the financial crisis of 2008 .

\section{Conclusion}

We have introduced the Joint Independent Metropolis-Hastings (JIMH) algorithm for the estimation of nonlinear non-Gaussian state space models with a Gaussian signal. We have concentrated on a univariate signal constructed out of a possibly high-dimensional state vector. We can conclude that the JIMH method is a computationally efficient alternative to competing MCMC methods such as the adaptive particle independent MetropolisHastings method. In a Monte Carlo study, we have shown that our method outperforms competing methods in terms of efficiency and computation time. An interesting extension for future research is to explore the sampling of state paths with the backward smoothing algorithm described in Lindsten and Schon (2012). This will relax the requirement of a Gaussian transition density, but may come at higher computational costs. 


\section{Acknowledgements}

A preliminary version of this paper was presented at ESOBE 2012 in Vienna, ERCIM 2012 in Oviedo, and the 3rd Humboldt-Copenhagen Conference on Financial Econometrics in Berlin in 2013. We are indebted to several participants at these meetings for constructive comments. André Lucas, Lennart Hoogerheide and István Barra thank the Dutch National Science Foundation (NWO) for financial support.

\section{References}

Andrieu, C., Doucet, A., Holenstein, R., 2010. Particle Markov chain Monte Carlo methods. Journal of Royal Statistical Society Series B 72, 269-342.

Azizpour, S., Giesecke, K., Schwenkler, G., 2010. Exploring the sources of default clustering, Working Paper, Stanford University.

Chan, J., Strachan, R., 2012. Estimation in non-linear non-Gaussian state space models with precision-based methods, CAMA Working Paper.

de Jong, P., Shephard, N., 1995. The simulation smoother for time series models. Biometrika 82, 339-350.

Doucet, A., de Freitas, N., Gordon, N. (Eds.), 2001. Sequential Monte Carlo Methods in Practice. Springer Verlag, New York.

Doucet, A., Pitt, M., Kohn, R., 2012. Efficient implementation of MCMC when using an unbiased likelihood estimator, Working Paper.

Duffie, D., Eckner, A., Horel, G., Saita, L., 2009. Frailty correlated default. Journal of Finance 64, 2089-2123.

Duffie, D., Saita, L., Wang, K., 2007. Multi-period corporate default prediction with stochastic covariates. Journal of Financial Economics 83 (3), 635-665.

Durbin, J., Koopman, S. J., 1997. Monte Carlo maximum likelihood estimation for nonGaussian state space models. Biometrika 84, 669-684.

Durbin, J., Koopman, S. J., 2002. A simple and efficient simulation smoother for state space time series models. Biometrika 89, 603-616.

Flury, T., Shephard, N., 2011. Bayesian inference based only on simulated likelihoood. Econometric Theory 27, 933-956.

Giordani, P., Kohn, R., 2010. Adaptive independent Metropolis-Hastings by fast estimation of mixtures of normals. Journal of Computational and Graphical Statistics 19, 243-259. 
Gordon, N., Salmond, D. J., Smith, A. F. M., 1993. A novel approach to non-linear and non-Gaussian Bayesian state estimation. IEE-Proceedings F 140, 107-113.

Hastings, W. K., 1970. Monte Carlo sampling methods using Markov chains and their applications. Biometrika 57, 97-109.

Hoogerheide, L. F., Opschoor, A., van Dijk, H. K., 2012. A class of adaptive importance sampling weighted EM algorithms for efficient and robust posterior and predictive simulation. Journal of Econometrics 171 (2), 101-120.

URL http://dx.doi.org/10.1016/j.jeconom.2012.06.011

Kim, S., Shephard, N., Chib, S., 1998. Stochastic volatility: Likelihood inference and comparison wih ARCH models. Review of Economic Studies 65, 361-393.

Koopman, S. J., Lucas, A., Monteiro, A., 2008. The multi-state latent factor intensity model for credit rating transition. Journal of Econometrics 142, 399-424.

Koopman, S. J., Lucas, A., Scharth, M., 2011. Numerically accelerated importance sampling for nonlinear non-Gaussian state space models, Tinbergen Institute Discussion Paper 2011-057/4.

Kullback, S., Leibler, R. A., 1951. On information and sufficiency. The Annals of Mathematical Statistics 22, 79-86.

Lando, D., Nielsen, M. S., 2010. Correlation in corporate defaults: contagion or conditional independence? Journal of Financial Intermediation 19, 355-372.

Lindsten, F., Schon, T. B., 2012. On the use of backward simulation in particle Markov chain Monte Carlo methods.

McCausland, W. J., 2012. The HESSIAN method: Highly efficient simulation smoothing, in a nutshell. Journal of Econometrics 168 (2), 189-206.

Metropolis, N., Rosenbluth, A. W., Rosenbluth, M. N., Teller, A. H., Teller, E., 1953. Equations of state calculations by fast computing machines. Journal of Chemical Physics 21, 1087-1092.

Pitt, M. K., Shephard, N., 1999. Filtering via simulation: auxiliary particle filter. Journal of the American Statistical Association 94, 590-599.

Pitt, M. K., Silva, R. S., Giordani, P., Kohn, R., 2012. On some properties of Markov chain Monte Carlo simulation methods based on the particle filter. Journal of Econometrics 171 (2), 134-151.

Richard, J., Zhang, W., 2007. Efficient high-dimensional importance sampling. Journal of Econometrics 141, 1385-1411.

Roberts, G. O., Rosenthal, J. S., 2009. Examples of adaptive MCMC. Journal of Computational and Graphical Statistics 18, 349-367.

Scharth, M., 2012. Essays on Monte Carlo methods for state space models. Ph.D. thesis, 
VU University Amsterdam.

Shephard, N., 2005. Stochastic Volatility: Selected Readings. Oxford University Press.

Shephard, N., Pitt, M. K., 1997. Likelihood analysis of non-Gaussian measurement time series. Biometrika 84, 653-667.

Zeevi, A. J., Meir, R., 1997. Density estimation through convex combination of densities; approximation and estimation bounds. Neural Networks 10, 99-106.

\section{A The Expectation and Maximization steps in MitISEM}

We want to maximize the weighted log-density

$$
\frac{1}{N} \sum_{j=1}^{N} w^{(j)} \log q_{\zeta}\left(\theta^{(j)} \mid y\right)
$$

where the weight $w^{(j)}$ is the ratio of the target density kernel and the candidate density from which the $d$-dimensional vector draws $\theta^{(j)}$ have been simulated, with weight $w^{(j)}$ and where $q_{\zeta}\left(\theta^{(j)} \mid y\right)$ is a mixture of $H$ Student's $t$-densities. The target density kernel is either the marginal posterior density kernel of $\theta$ or the joint posterior density kernel of $\theta$ and the signal $x$; in the latter case the candidate density is the joint candidate density for $\theta^{(j)}$ and $x^{(j)}$ where $x^{(j)}$ has been simulated conditionally on $\theta^{(j)}$. We can write the mixture of Student's $t$-densities using a latent variable representation where $z^{(j)}$ is a latent $H$ dimensional vector consisting of $H-1$ zeros and one element $z_{h}^{j}=1$ that indicates that the draw $\theta^{(j)}$ belongs to the $h$-th Student's $t$-distribution. The mixing weight is $\operatorname{Pr}\left[z_{h}^{j}=1\right]=\eta_{h}$, and

$$
\theta^{(j)} \sim \mathrm{N}(\mu, \Sigma), \quad \mu=\sum_{i=1}^{H} z_{h}^{j} \mu_{h}, \quad \Sigma=\sum_{i=1}^{H} z_{h}^{j} \kappa_{h}^{j} \Sigma_{h},
$$

where $\mu_{h}$ and $\Sigma_{h}$ are the mode vector and scale matrix of the $h$-th Student's $t$-distribution, and where the random variable $\kappa_{h}^{j}$ has an Inverse-Gamma distribution

$$
\kappa_{h}^{j} \sim I G\left(\nu_{h} / 2, \nu_{h} / 2\right)
$$

where $\nu_{h}$ is the degrees of freedom parameter of the $h$-th Student's $t$-distribution.

The Expectation-Maximization (EM) algorithm proceeds with iterations $L(L=$ $1,2, \ldots)$, which consist of an expectation and maximization step, until it has converged to a (local) optimum. In the expectation step of iteration $L$ the conditional expectations 
of the expressions involving the latent variables $z^{j}$ and $\kappa^{j}$ that occur in the log-density, given the draws $\theta^{(j)}$ and $\zeta=\zeta^{(L-1)}=\left\{\mu_{h}, \Sigma_{h}, \nu_{h}, \eta_{h} ; h=1, \ldots, H\right\}$, the parameters from the previous EM iteration $(L-1)$, are the following:

$$
\tilde{z}_{h}^{j} \equiv \mathrm{E}\left[z_{h}^{j} \mid \theta^{(j)}, \zeta=\zeta^{(L-1)}\right]=\frac{t\left(\theta^{(j)} \mid \mu_{h}, \Sigma_{h}, \nu_{h}\right) \eta_{h}}{\sum_{i=1}^{H} t\left(\theta^{(j)} \mid \mu_{i}, \Sigma_{i}, \nu_{i}\right) \eta_{i}},
$$

where $t(\cdot \mid \mu, \Sigma, \nu)$ is a Student's $t$-density with mode $\mu$, scale matrix $\Sigma$ and degree of freedom $\nu$,

$$
\begin{aligned}
{\widetilde{z / \kappa_{h}}}^{j} & \equiv \mathrm{E}\left[\frac{z_{h}^{j}}{\kappa_{h}^{j}} \mid \theta^{(j)}, \zeta=\zeta^{(L-1)}\right]=\tilde{z}_{h}^{j} \frac{d+\nu_{h}}{\rho_{h}^{j}+\nu_{h}} \\
\xi_{h}^{i} & \equiv \mathrm{E}\left[\log \kappa_{h}^{j} \mid \theta^{(j)}, \zeta=\zeta^{(L-1)}\right]= \\
& =\left[\log \left(\frac{\rho_{h}^{j}+\nu_{h}}{2}\right)-\psi\left(\frac{d+\nu_{h}}{2}\right)\right] \tilde{z}_{h}^{j}+\left[\log \left(\frac{\nu_{h}}{2}\right)-\psi\left(\frac{\nu_{h}}{2}\right)\right]\left(1-\tilde{z}_{h}^{j}\right), \\
\delta_{h}^{i} & \equiv \mathrm{E}\left[\frac{1}{\kappa_{h}^{j}} \mid \theta^{(j)}, \zeta\right]={\widetilde{z / \kappa_{h}}}^{j}+\left(1-\tilde{z}_{h}^{j}\right)
\end{aligned}
$$

with $\rho_{h}^{j} \equiv\left(\theta^{(j)}-\mu_{h}\right)^{\prime} \Sigma_{h}^{-1}\left(\theta^{(j)}-\mu_{h}\right)$, and $\psi($.$) is the digamma function.$

The maximization step of iteration $L$ consists of the following updates

$$
\begin{gathered}
\mu_{h}^{(L)}=\left[\sum_{j=1}^{N} w^{(j)}{\widetilde{z / \kappa_{h}}}_{h}^{-1}\left[\sum_{j=1}^{N} w^{(j)} \theta^{(j)}{\widetilde{z / \kappa_{h}}}_{h}\right],\right. \\
\Sigma_{h}^{(L)}=\frac{\sum_{j=1}^{N} w^{(j)}\left(\theta^{(j)}-\mu_{h}^{(L)}\right)\left(\theta^{(j)}-\mu_{h}^{(L)}\right)^{\prime} z / \kappa_{h}^{j}}{\sum_{j=1}^{N} w^{(j)} \tilde{z}_{h}^{j}}, \\
\eta_{h}^{(L)}=\frac{\sum_{j=1}^{N} w^{(j)} \tilde{z}_{h}^{j}}{\sum_{j=1}^{N} w^{(j)}} .
\end{gathered}
$$

Finally $\nu_{h}^{(L)}$ is obtained by solving the first-order condition

$$
-\psi\left(\nu_{h} / 2\right)+\log \left(\nu_{h} / 2\right)+1-\frac{\sum_{j=1}^{N} w^{(j)} \xi_{h}^{j}}{\sum_{j=1}^{N} w^{(j)}}-\frac{\sum_{j=1}^{N} w^{(j)} \delta_{h}^{j}}{\sum_{j=1}^{N} w^{(j)}}=0
$$

for $\nu_{h}$. For more details we refer to Hoogerheide et al. (2012). 


\section{B NAIS}

We can write the likelihood of the state space model given by (1) and (2) as

$$
L(y \mid \theta)=\int \frac{p(x, y \mid \theta)}{q(y \mid x, \theta)} q(y \mid x, \theta) \mathrm{d} x=q(y \mid \theta) \int \omega(x, y \mid \theta) q(y \mid x, \theta) \mathrm{d} x
$$

where $x=\left(x_{1}, \ldots, x_{T}\right)^{\prime}$, with $x_{t}=c_{t}+Z_{t} \alpha_{t}$ being the signal at time $t$ for $t=1, \ldots, T$, and where

$$
\omega(x, y \mid \theta) \equiv p(y \mid x, \theta) / q(y \mid x, \theta)
$$

The Gaussian importance or proposal density can be written as

$$
q\left(y_{t} \mid x_{t}, \theta\right)=\exp \left\{a_{t}+b_{t}^{\prime} x_{t}-\frac{1}{2} x_{t}^{\prime} C_{t} x_{t}\right\}
$$

where $a_{t}, b_{t}$ and $C_{t}$ depend on the observations $y$ and the parameters in $\theta$ for $t=1, \ldots, T$. The importance density at time $t$ is effectively determined by $b_{t}$ and $C_{t}$ as the constant $a_{t}$ is chosen such that the density integrates to one. This restriction and $a_{t}$ do not play a role when we represent the Gaussian importance density as the smoothed density in the linear Gaussian state space model with its observation equation given by

$$
y_{t}^{*}=x_{t}+\varepsilon_{t}, \quad \varepsilon \sim \mathrm{N}\left(0, C_{t}^{-1}\right), \quad t=1, \ldots, T,
$$

where $y_{t}^{*}=C_{t}^{-1} b_{t}$ for $t=1, \ldots, T$ and the transition density given in equation (2).

To formulate an effective importance density we choose its parameters, as collected in $\chi=\left\{b_{1}, \ldots, b_{T}, C_{1}, \ldots, C_{T}\right\}$, by minimizing a conveniently chosen metric associated with the importance sample variation, that is

$$
\min _{\chi_{t}} \int \lambda^{2}\left(x_{t}, y_{t} \mid \theta\right) \omega\left(x_{t}, y_{t} \mid \theta\right) q\left(x_{t} \mid y^{*}, \theta\right) \mathrm{d} x_{t},
$$

for every $t$, where

$$
\lambda\left(x_{t}, y_{t} \mid \theta\right)=\log p\left(y_{t} \mid x_{t}, \theta\right)-\log q\left(y_{t}^{*} \mid x_{t}, \theta\right)-\lambda_{0 t} .
$$

We can rewrite the minimization as

$$
\min _{\chi_{t}} \sum_{j=1}^{M} \lambda^{2}\left(\tilde{x}_{t j}, y_{t} \mid \theta\right) \omega_{t j}, \quad \omega_{t j}=q\left(\tilde{x}_{t j} \mid y^{*}, \theta\right) \omega\left(\tilde{x}_{t j}, y_{t} \mid \theta\right) h\left(z_{j}\right) e^{z_{j}^{2}},
$$

with $\tilde{x}_{t j}=\hat{x}_{t}+V_{t}^{1 / 2} z_{j}$, for $j=1, \ldots, M$, and

$$
q\left(\tilde{x}_{t j} \mid y^{*}, \theta\right)=\exp \left\{-\frac{1}{2} z_{j}^{2}\right\} / \sqrt{2 \pi}, \quad t=1, \ldots, T,
$$


where $\hat{x}_{t}$ is the smoothed signal, $V_{t}$ is smoothed signal variance and $z_{j}$ are the abscissa designated by the Gauss-Hermite quadrature. The minimization is carried out iteratively. First, for a given $\chi$ we obtain $\hat{x}_{t}$ and $V_{t}$ for $t=1, \ldots, T$ from the linear Gaussian state space model in (A14). Second, we obtain the optimal $\chi_{t}=\left\{C_{t}, b_{t}\right\}$ for $t=1, \ldots, T$ by a weighted least squares computation with "dependent" variable $\log p\left(y_{t} \mid \tilde{x}_{t j}, \theta\right)$ and "explanatory variables" $\tilde{x}_{t j}$ and $\tilde{x}_{t j}^{2}$. We iterate these steps until convergence of $\chi$. For a more detailed discussion, we refer to Koopman et al. (2011).

\section{Adaptive random-walk Metropolis-Hastings}

Roberts and Rosenthal (2009) propose an adaptive random walk Metropolis-Hastings algorithm, with a proposal of the following form

$$
q_{n}\left(\theta ; \theta_{n-1}\right)=\omega_{1 n} \phi_{d}\left(\theta ; \theta_{n-1}, \kappa_{1} \Sigma_{1}\right)+\omega_{2 n} \phi_{d}\left(\theta ; \theta_{n-1}, \kappa_{2} \Sigma_{2 n}\right)
$$

where $\phi_{d}(\theta ; \hat{\theta}, \Sigma)$ is a $d$ dimensional multivariate normal density with mean $\hat{\theta}$ and covariance matrix $\Sigma$. We set $\omega_{1 n}=1$ until $n>n_{0}$, and $\omega_{1 n}=0.05$ afterwards. The scalars $\kappa_{1}=0.1^{2} / d$ and $\kappa_{2}=2.38^{2} / d$ and $\Sigma_{1}=I_{d}$ are constant throughout the procedure, while $\Sigma_{2 n}$ covariance matrix is estimated using the first $n-1$ iterates.

\section{Adaptive mixture of normals}

Giordani and Kohn (2010) and Pitt et al. (2012) suggest an adaptive mixture of normals proposal, which has the form

$$
q_{n}(\theta)=\sum_{k=1}^{4} \omega_{k n} q_{k n}(\theta) \quad \omega_{k n} \leq 0, \quad \text { for } \quad k=1, \ldots, 4 \quad \text { and } \quad \sum_{k=1}^{4} \omega_{k n}=1,
$$

at iteration $n$. The adaptation has two stages. We start the first stage with setting $\omega_{1 n}=0.8, \omega_{2 n}=0.2$ and we use a Gaussian density for $q_{1 n}$ with mean equal to the simulated maximum likelihood estimates and variance equal to minus the inverse Hessian at the mean. Moreover we set $q_{2 n}$ as a heavy tailed version of $q_{1 n}$ by setting the covariance matrix 15 times the covariance matrix of $q_{1 n}$. After $5 d$ accepted draws (where $d$ is equal to the dimension of $\theta$ ) we set $q_{3 n}$ and $q_{4 n}$ and we change the component weights in (A20). $q_{3 n}$ is obtained as a mixture of normals using k-means clustering on the previous draws. $q_{4 n}$ is the fat tailed version of $q_{3 n}$, it has the same means and mixture probabilities as 
$q_{3 n}$ but the covariance matrices are multiplied by 20 . The new weights are the following $\omega_{1 n}=0.15, \omega_{2 n}=0.05, \omega_{3 n}=0.7, \omega_{4 n}=0.1$. In the rest of the first stage we update $q_{3 n}$ at predetermined updating times or after rejecting 10 candidate draws in a row. We always set $q_{4 n}$ to be the fat tailed version of $q_{3 n}$. The first stage ends if the minimal acceptance rate (i.e., the conditional acceptance probability in the $\mathrm{MH}$ algorithm) in the last 1000 draws is above 0.02. After the first stage we set $q_{1 n}=q_{3 n}$, i.e., the last version of the mixture of normals, and $q_{2 n}$ is again the fat tailed version of the new $q_{1 n}$. In the second stage we only update at predetermined updating times. 